\title{
Tetrandrine blocks autophagic flux and induces apoptosis via energetic impairment in cancer cells
}

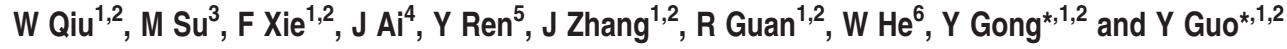

Lysosomes are acidic organelles that have a crucial role in degrading intracellular macromolecules and organelles during the final stage of autophagy. Tetrandrine (Tet), a bisbenzylisoquinoline alkaloid, was reported as an autophagy activator. Here, in contrast with previous studies, we show that Tet is a potent lysosomal deacidification agent and is able to block autophagic flux in the degradation stage. Single-agent Tet induces significant apoptosis both in vitro and in xenograft models. In the presence of Tet, apoptosis was preceded by a robust accumulation of autophagosomes and an increased level of microtubule-associated protein 1 light chain 3, type II (LC3-II). However, Tet increased the level of sequestosome 1 and decreased the turnover of LC3, indicating the blockade of autophagic flux in the degradation stage. As blockade of autophagic flux decreases the recycling of cellular fuels, Tet reduces the uptake of glucose in cancer cells. These effects lead to insufficient substrates for tricarboxylic acid (TCA) cycle and impaired oxidative phosphorylation. Blunting autophagosome formation using 3-methyladenine or genetic knockdown of Beclin-1 failed to rescue cells upon Tet treatment. By contrast, addition of methyl pyruvate to supplement TCA substrates protected Tet-treated tumor cells. These results demonstrate that energetic impairment is required in Tet-induced apoptosis. Tet, as a potent lysosomal inhibitor, is translatable to the treatment of malignant tumor patients.

Cell Death and Disease (2014) 5, e1123; doi:10.1038/cddis.2014.84; published online 13 March 2014

Subject Category: Cancer

Macroautophagy (hereafter autophagy) is a lysosomedependent process by which cytoplasmic elements, including long-lived cytoplasmic proteins, glycogen, and damaged organelles, are degraded. The entire process of autophagy, known as autophagic flux, includes the delivery of cargo to lysosomes, the breakdown of cargo, and releasing of molecules into the cytosol. ${ }^{1}$ As the number of autophagosomes is frequently used to evaluate autophagy, the accumulation of them in the cytosol reflects increased formation of autophagosomes or reduced turnover of them. For the latter, despite the fact that the number of autophagosomes is increased, the autophagic flux is actually blocked. Therefore, it is critical to distinguish between the two possibilities for accurately assessing the changes of autophagic flux under certain treatment.

Tetrandrine (Tet) is a natural product isolated from the root of Stephania tetrandra S. Moore. ${ }^{2}$ Among its clinical applications, Tet has been used in the treatment of rheumatoid arthritis, sepsis, endotoxin-induced uveitis, silicosis, and hypertension. ${ }^{3-6}$ In these years, numerous studies have focused on the antitumor activity of Tet, with several of them showing that Tet contributes to the reversal of multidrug resistance in cancer cells and enhances their radiosensitivity. ${ }^{7-13}$ More recently, Tet was shown to activate autophagy in human hepatocellular carcinoma (HCC) cells. ${ }^{14}$ Despite Tet treatment inducing the formation of acidic autophagolysosome vacuoles and increased levels of microtubule-associated protein 1 light chain 3 , type II (LC3-II),${ }^{14}$ the events of autophagy in the degradation stage as well as the role of autophagy in Tet-induced cell death has never been investigated before.

Tet belongs to the bisbenzylisoquinoline alkaloid family. ${ }^{2}$ The structure of Tet contains two nitrogen atoms, which make the solution of it alkaline. Most weak bases, such as chloroquine (CQ) and its derivatives, can be protonated and accumulates in lysosomes, resulting in the deacidification of their lumens. ${ }^{15,16}$ The lumen of the lysosome contains $\sim 60$ types of soluble hydrolases. ${ }^{17}$ These enzymes are active in acidic environments and, as such, are responsible for the lysosomal hydrolysis of many different substrates, including during autophagy. ${ }^{17}$ Autophagic flux can be blocked when the acidity of lysosome is reduced by CQ or other weak bases. On the basis of the above, we hypothesized that Tet could neutralize lysosomal acidity and eventually block the

\footnotetext{
${ }^{1}$ Department of Urology, Peking University First Hospital and the Institute of Urology, Peking University, Beijing 100034, China; ${ }^{2}$ National Urological Cancer Center, Beijing 100034, China; ${ }^{3}$ Sino-German Laboratory for Molecular Medicine, State Key Laboratory of Cardiovascular Disease, FuWai Hospital \& Cardiovascular Institute, Chinese Academy of Medical Sciences, Peking Union Medical College, Beijing 100037, China; ${ }^{4}$ Department of Urology, University of Pittsburgh, Pittsburgh, PA 15232, USA; ${ }^{5}$ Lab of Electron Microscopy, Peking University First Hospital, Beijing 100034, China and ${ }^{6}$ Department of Urology, Provincial Hospital affiliated to Shandong University, Jinan 250021, China

${ }^{*}$ Corresponding author: Y Gong or Y Guo, Department of Urology, Peking University First Hospital and the Institute of Urology, Peking University, No. 8, Xishiku Street, Xicheng District, Beijing 100034, China. Tel: +86 108357 2418; Fax: +86 106655 1028; E-mail: GYL07@ sina.com or yqgong@ bjmu.edu.cn

Keywords: tetrandrine; autophagy; lysosomes; apoptosis; metabolism

Abbreviations: Tet, tetrandrine; $\mathrm{CQ}$, chloroquine; PARP, polyADP-ribosepolymerase; $\Delta \Psi \mathrm{m}$, mitochondrial membrane potential; TCA, tricarboxylic acid; 3-MA, 3methyladenine; LC3, microtubule-associated protein 1 light chain 3; SQSTM1/p62, sequestosome 1; OXPHOS, oxidative phosphorylation; OCR, oxygen consumption rate; TUNEL, terminal deoxynucleotidyl transferase-dUTP nick-end labeling; MP, methyl pyruvate; AMPK $\alpha$, AMP-activated protein kinase $\alpha$; ROS, reactive oxygen species; 2-NBDG, 2-(N-(7-nitrobenz-2-oxa-1,3-diazol-4-yl)amino)-2-deoxyglucose

Received 21.10.13; revised 19.12.13; accepted 07.1.14; Edited by GM Fimia
} 
autophagic flux. In the present study, we specially focus on the effects of Tet on lysosomal acidification and autophagic flux in tumor cells. We found that Tet is a potent lysosomal deacidification agent that blocks autophagic flux. Moreover, Tet impairs the generation of substrates for tricarboxylic acid (TCA) cycle, resulting in energy depletion and apoptosis in tumor cells. Our results provide evidence for the clinical use of Tet as a lysosome inhibitor in treating malignant tumors.

\section{Results}

Tet induces apoptosis by activating caspase-3 in tumor cells in vitro. Previous studies reported that Tet is able to inhibit cell growth in tumor cells. In order to compare whether Tet has same effects on different cell lines, CCK-8 assays were conducted using three human cancer cell lines: PC-3, 786-O, and PANC-1. In cells treated with 10 or $20 \mu \mathrm{M}$ of Tet for 12,24 , or $48 \mathrm{~h}$, viability significantly decreased as the Tet dose or exposure time increased (Figure 1a). The inhibitory concentration 50 of Tet in the 11 established tumor cell lines, determined after $48 \mathrm{~h}$ of incubation with the drug, ranged from 2.62 to $8.03 \mu \mathrm{M}$ (Figure $1 \mathrm{~b}$ ). These results suggested that Tet had broad antitumor effects in various cell lines.

Apoptosis in Tet-treated cells was followed using Annexin V-FITC and propidium iodide (PI) fluorescence staining. As shown in Figure 1c, Tet induced a moderate rate of apoptosis at $12 \mathrm{~h}$, as indicated by an increased proportion of Annexin V-positive cells (Supplementary Figure 1). However, high levels of late-stage of apoptosis (Annexin V-positive/PI-positive cells) could be obtained in cells treated with $10 \mu \mathrm{M}$ Tet for 24 and $48 \mathrm{~h}$ (Figure 1c). Because little is known about the effects of Tet on primary cultured tumor cells, we isolated tumor cells from prostate and renal cancer patients to evaluate the antitumor effects of Tet. Both of the cultured primary prostate and renal cancer cells showed increased apoptotic rate, similar as in cell lines (Figure $1 \mathrm{~d}$ ). We next measured $\Delta \Psi m$ with JC-1 staining, as a decrease in $\Delta \Psi m$ often accompanies apoptosis. Indeed, in $\mathrm{PC}-3$ and $786-\mathrm{O}$ cells treated with Tet for 12,24 , and $48 \mathrm{~h}, \Delta \Psi \mathrm{m}$ decreased significantly (Figure 1e). In addition, the cleavage of caspase-3 and of its substrate polyADP-ribosepolymerase (PARP) was determined. The results showed that, as the Tet exposure time increased, cleavage of caspase-3 and PARP in PC-3, 786-O, and PANC-1 cells increased as well (Figure 1f).

As caspase-dependent apoptosis can be inhibited by a pan-caspase inhibitor Z-VAD-FMK, we next determined Tet-induced cell death in the presence of the inhibitor. As expected, co-treatment of PC-3 and 786-O cells with Z-VAD-FMK partially protected against Tet-induced cell death (Supplementary Figure 2). In contrast, Necrostatin-1, a necroptosis inhibitor, failed to attenuate Tet-induced cell death (Supplementary Figure 3). These results suggested that Tet is able to induce apoptosis rather than necroptosis in tumor cells.

Tet inhibits tumor growth by inducing apoptosis in PC-3 tumor xenografts. We next sought to investigate whether Tet exerts antitumor effects in vivo. Because the effects of Tet on prostate cancer, especially androgen-independent prostate cancers, have never been reported previously, we performed the in vivo studies with PC-3 tumor xenograft models. Nude mice bearing tumor xenografts were treated with either vehicle alone or Tet $(60 \mathrm{mg} / \mathrm{kg})$, administered as an intraperitoneal injection once every other day for 27 days. Tumor sizes were measured on each injection day. During the 27 days of Tet treatment, the mice well tolerated the drug without a loss of body weight. The results mirrored those of the in vitro studies, with Tet treatment significantly decreasing tumor growth (Figure 2a). At the end of the treatment period, tumors in the vehicle group had reached an average size of $543.03 \mathrm{~mm}^{3}$ compared with $268.2 \mathrm{~mm}^{3}$ in the Tet-treated group (Figure $2 \mathrm{~b}$ ). Consistent with the changes in tumor volume, the average tumor weight was significantly reduced in the Tet-treated group (Figure 2c) (0.21 versus $0.08 \mathrm{~g}$ in the vehicle group; $P<0.05)$. TUNEL assays were performed to detect apoptotic cells in the prostate cancer xenografts. As shown in Figure 2d, the number of apoptotic cells was significantly higher in the xenografts of the Tet-treated group than in those of the vehicle group (18.09\% versus $3.53 \% ; P<0.01)$. Taken together, our results clearly show that Tet is able to inhibit prostate cancer growth in vivo.

Tet increases intracellular autophagosomes in vitro and in vivo. In previous studies, Tet was shown to induce both apoptosis and autophagy according to Tet's concentration in HCC cell lines. ${ }^{14,18}$ To test our hypothesis, we should first confirm whether Tet is able to increase autophagosome formation. Therefore, we observed the intracellular autophagic vacuoles under electron microscopy. Consistently, in Tettreated PC-3, 786-O cells, and prostate cancer xenografts, a greater number of autophagic vacuoles were observed (Figure 3a). We then transfected PC-3 and 786-O cells with the enhanced green fluorescent protein-microtubuleassociated protein 1 light chain 3 (EGFP-LC3) reporter, as a specific indicator of autophagic vacuoles. The redistribution of EGFP-LC3 from cytosol to autophagosome indicates the formation of autophagosomes. In the transfected cells treated with Tet $(10 \mu \mathrm{M})$ for $6 \mathrm{~h}$, significant punctate dots in Tet-treated PC-3 and 786-O cells were observed (Figure $3 b$ ). By contrast, control cells showed a relative diffuse green fluorescence in the cytosol. Similar results were obtained from stable EGFP-LC3-transformed HeLa cells (Supplementary Figure 4). These results demonstrated that Tet significantly increased the number of autophagosomes in tumor cells. We then measured the levels of LC3-II as an indicator of autophagosome formation. As expected, LC3-II levels in PC-3, 786-O, and PANC-1 cells were increased in a doseand time-dependent manner (Figure 3c; Supplementary Figure 5). Similar results were obtained with cultured primary prostate cancer cells, primary renal cancer cells, and prostate cancer xenografts (Figures $3 d-f$ ). Taken together, these data show that Tet robustly increases the number of autophagosomes in various tumor cells, before Tet induced apoptosis.

Tet inhibits autophagic flux by impairing clearance of autophagosomes. The number of autophagosomes in the cytosol can be increased by two different mechanisms: increased activation of autophagy by upstream processes and blockade of lysosomal degradation at a later stage. 

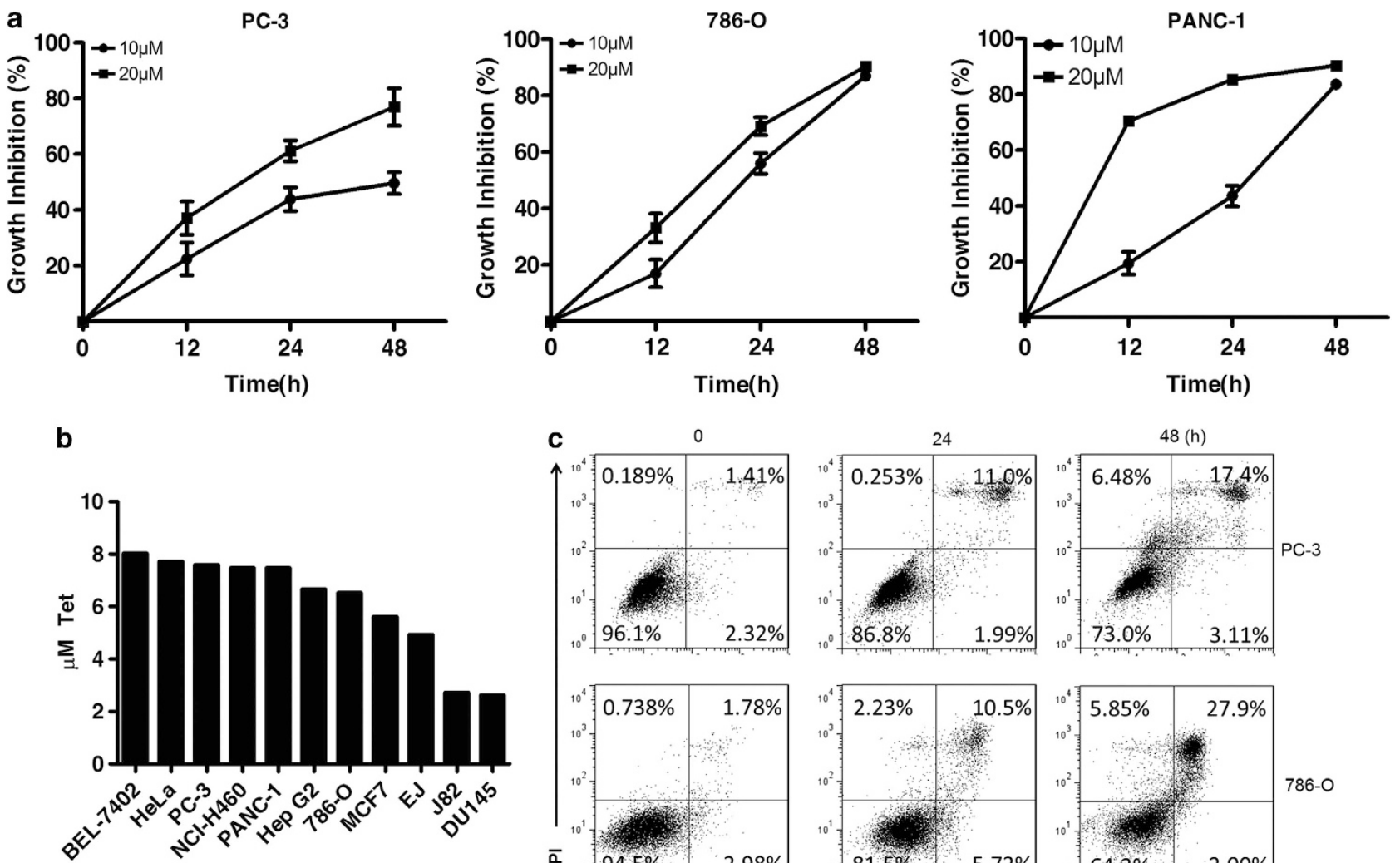

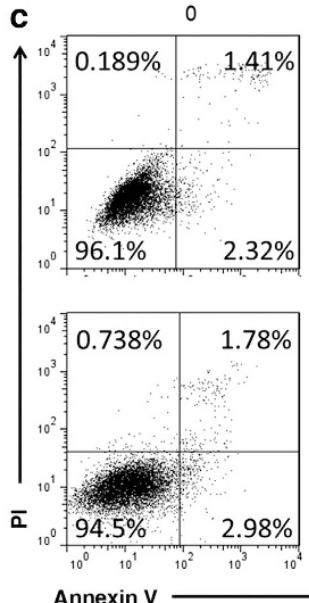

Annexin V

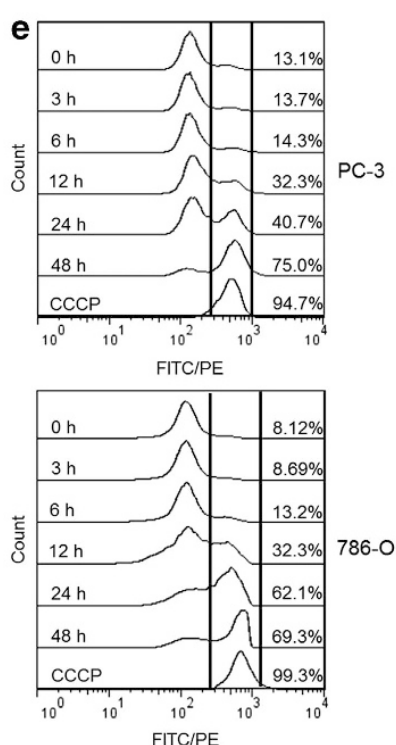

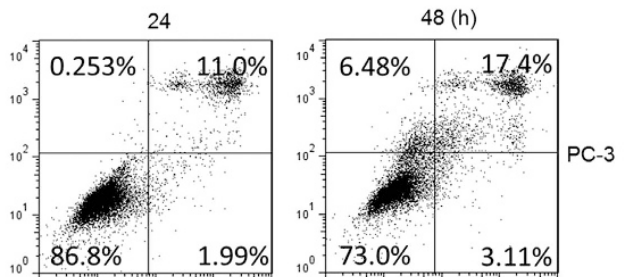
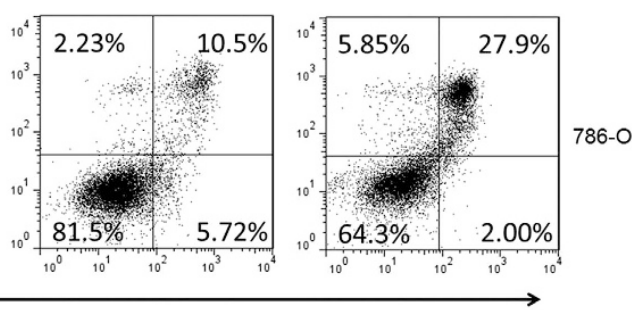

f

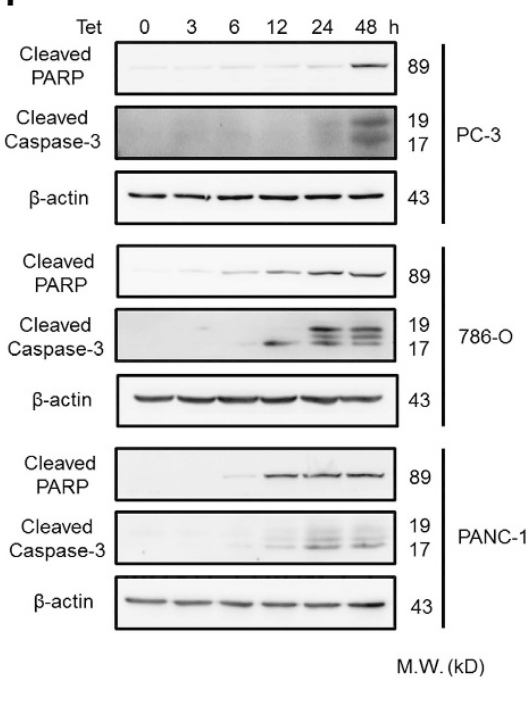

Figure 1 Tet induces apoptosis in multiple types of cancer cells in vitro. (a) PC-3, 786-0, and PANC-1 cells were treated with Tet $(10$ and $20 \mu \mathrm{M})$ for 12,24 , and $48 \mathrm{~h}$. After treatment, cell viability was determined using CCK-8 assays. The data are presented as the mean \pm S.D. of three independent experiments. (b) The $I_{50}$ of Tet after $48 \mathrm{~h}$, as determined in a panel of cancer cell lines. (c) PC-3 and 786-O cells were treated with Tet $(10 \mu \mathrm{M})$ for 24 or $48 \mathrm{~h}$, after which the cells were harvested, stained with Annexin V/ $\mathrm{PI}$, and examined by flow cytometry. (d) Primary prostate cancer cells and primary renal cancer cells treated with Tet $(10 \mu \mathrm{M})$ for $48 \mathrm{~h}$ were stained with Annexin V/PI and examined by flow cytometry. (e) The mitochondrial membrane potential of PC-3 and 786-0 cells treated with Tet $(10 \mu \mathrm{M})$ for the indicated times was measured by JC-1 staining. The uncoupler CCCP served as the positive control. (f) PC-3, 786-O, and PANC-1 cells were treated with Tet (10 $\mu \mathrm{M})$ for the indicated times after which cleaved PARP and cleaved caspase-3 were detected by western blotting. $\beta$-actin served as the loading control. The experiments were performed at least three times, with representative results shown in the figure 


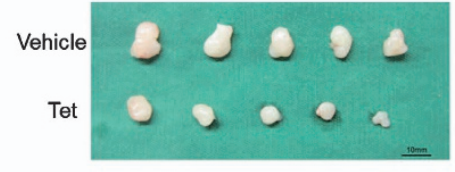

C

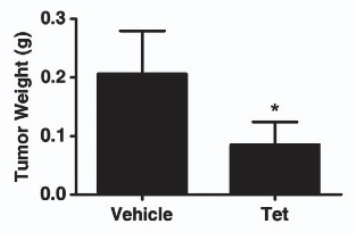

b

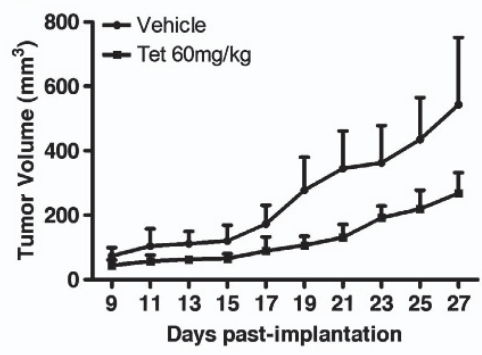

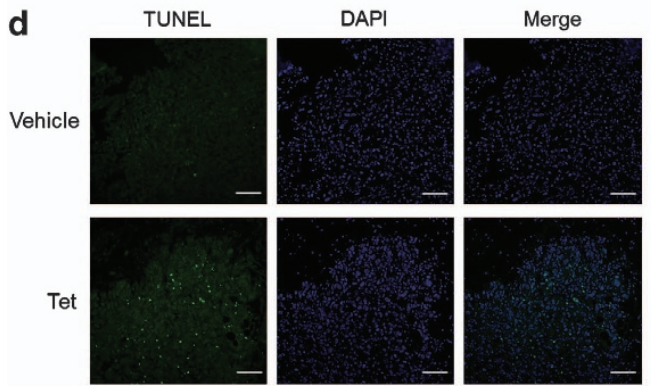

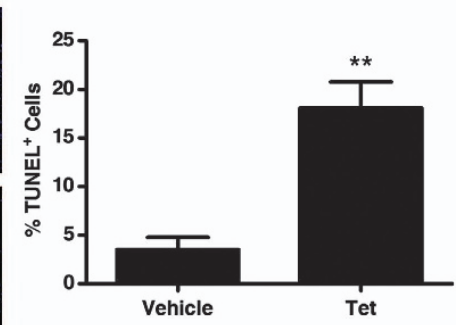

Figure 2 Tet suppresses tumor growth and induces apoptosis in mice bearing PC-3 xenografts. The mice were subcutaneously inoculated in the right flank with $3 \times 10^{6}$ PC-3 cells and randomly distributed into two groups (five per group): vehicle and $60 \mathrm{mg}$ Tet/kg body weight, injected intraperitoneally every two days for 27 days. (a) The appearance of prostate cancer xenografts of dissected tumors. (b) Tumor size was measured every other day. Tumor volume is expressed as the mean \pm S.D. (c) After 27 days, all the mice were killed, and the tumors were dissected and weighed. The results are presented as the mean \pm S.D.; ${ }^{*} P<0.05$. (d) Tumor tissues were fixed and subjected to TUNEL assays, examined with fluorescence microscopy (scale bars, $50 \mu \mathrm{m}$ ) (left), TUNEL-positive cells counted in five high-power fields per slide (right). Bars, S.D.; columns, mean; ${ }^{* *} P<0.01$

To discriminate between these two mechanisms in Tettreated cells in Tet-induced cell death, we measured SQSTM1/p62 expression in tumor cells challenged with Tet. SQSTM1/p62 is an ubiquitin-binding protein that is degraded by autophagy; ${ }^{19}$ when autophagy is inhibited, the degradation of this protein is lessened and it accumulates in the cytosol. In contrast, if the autophagic flux is activated, the degradation of this protein is accelerated and thus decreases its level in the cell. ${ }^{19}$ As seen in Figure 3c, SQSTM1/p62 levels increased in a concentration- and time-dependent manner in Tet-treated PC-3, 786-O, and PANC-1 cells. Similar results were obtained in cultured primary prostate cancer cells, primary renal cancer cells, and prostate cancer xenografts (Figures $3 d$, e and $\mathrm{g}$ ). These results therefore support the conclusion that the Tet-induced accumulation of autophagosomes reflects an inhibition of their degradation. This conclusion was supported by the results of LC3 turnover assays carried out as described by Mizushima et al. ${ }^{20}$ PC-3 and $786-\mathrm{O}$ cells were treated with Tet $(10 \mu \mathrm{M})$ in the presence or absence of $\mathrm{CQ}(10 \mu \mathrm{M})$ for $1 \mathrm{~h}$. To mimic autophagy induction, we treated cells with rapamycin $(200 \mathrm{nM})$ as a control. If the autophagy is induced, co-treatment with CQ will increase the LC3-II level. On the contrary, LC3-II level will not be affected in the presence of $C Q$. As expected, we observed that $C Q$ treatment had no effects on LC3-II levels in Tet-treated cells (Figure 4a). By contrast, in rapamycin-treated cells, co-treatment with $\mathrm{CQ}$ increased LC3-II levels (Figure 4a).

To provide more evidence that Tet blocks autophagic flux in tumor cells, we transfected PC-3 and 786-O cells with a plasmid encoding membrane-localized red fluorescent protein
(mRFP)-EGFP-LC3. Expression of this tandem fluorescenttagged LC3 (tfLC3) results in both green and red fluorescence (Figure 4b). ${ }^{21}$ EGFP fluorescence is easily quenched in a low-pH environment, whereas mRFP is more stable in an acidic environment. Colocalization of EGFP and mRFP fluorescence (merged as yellow) indicates compartments that are not fused with acidic lysosomes. Therefore, both yellow and red punctate fluorescence will increase in the case of autophagy activation, whereas blockade in the degradation stage results in only yellow punctate fluorescence. ${ }^{21}$ After treatment of the cells with Tet $(10 \mu \mathrm{M})$ for $3 \mathrm{~h}$, yellow punctate fluorescence was markedly increased, indicating a blockade of autophagy in Tet-treated cells (Figures $4 b$ and c). Similarly, in cells treated with bafilomycin A1 (200 nM) for $1 \mathrm{~h}$, which blocks autophagosome-autolysosome fusion, yellow punctate fluorescence increased, whereas rapamycin (200 nM), an activator of autophagy, caused an increase in both red and yellow punctate fluorescence (Figures $4 b$ and $c$ ). Taken together, these results provide strong evidence that Tet impairs the clearance of autophagosomes, resulting in their accumulation in the cytosol. This conclusion supports that Tet is a potent autophagy inhibitor rather than an activator in tumor cells.

Tet decreases the acidity of lysosome in tumor cells. The role of the lysosome in autophagy is to degrade cargos in the autophagosome. A low $\mathrm{pH}$ in the lysosome is required for the activity of lysosomal enzymes. ${ }^{17}$ Weak bases such as $\mathrm{CQ}$ block autophagy by elevating lysosomal $\mathrm{pH}$. On the basis of our finding that Tet impaired the clearance of autophagosomes, we speculated that lysosomal function might 

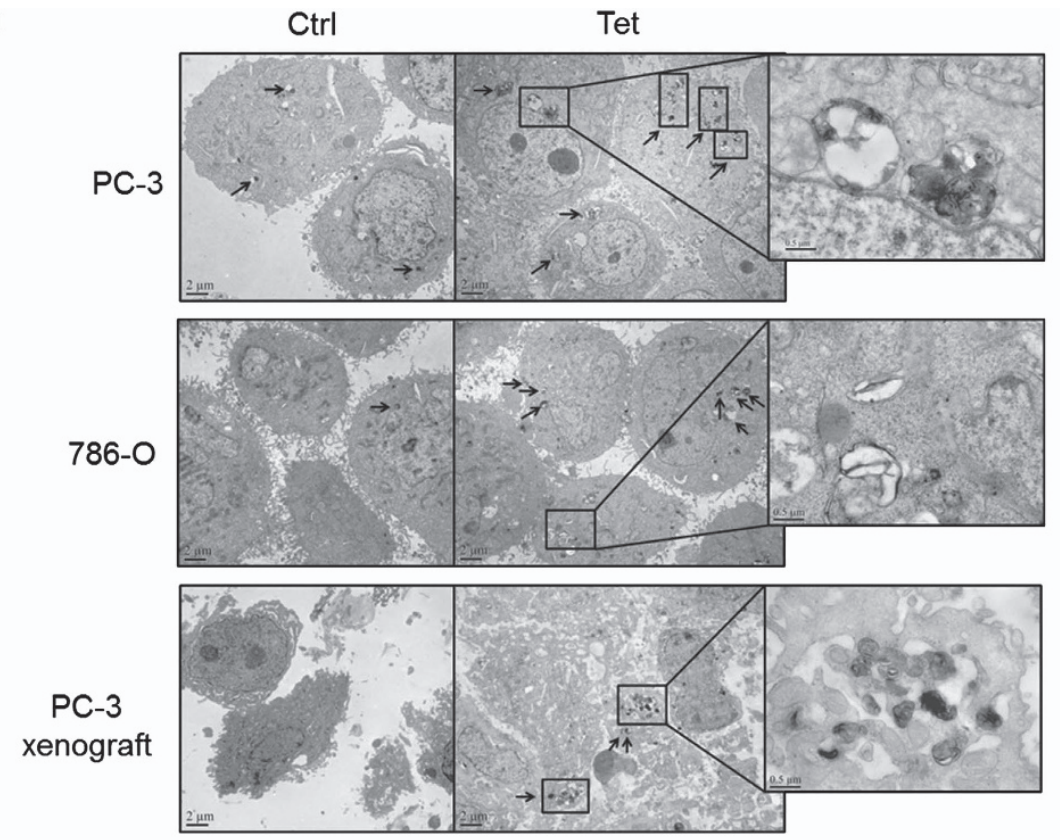

C

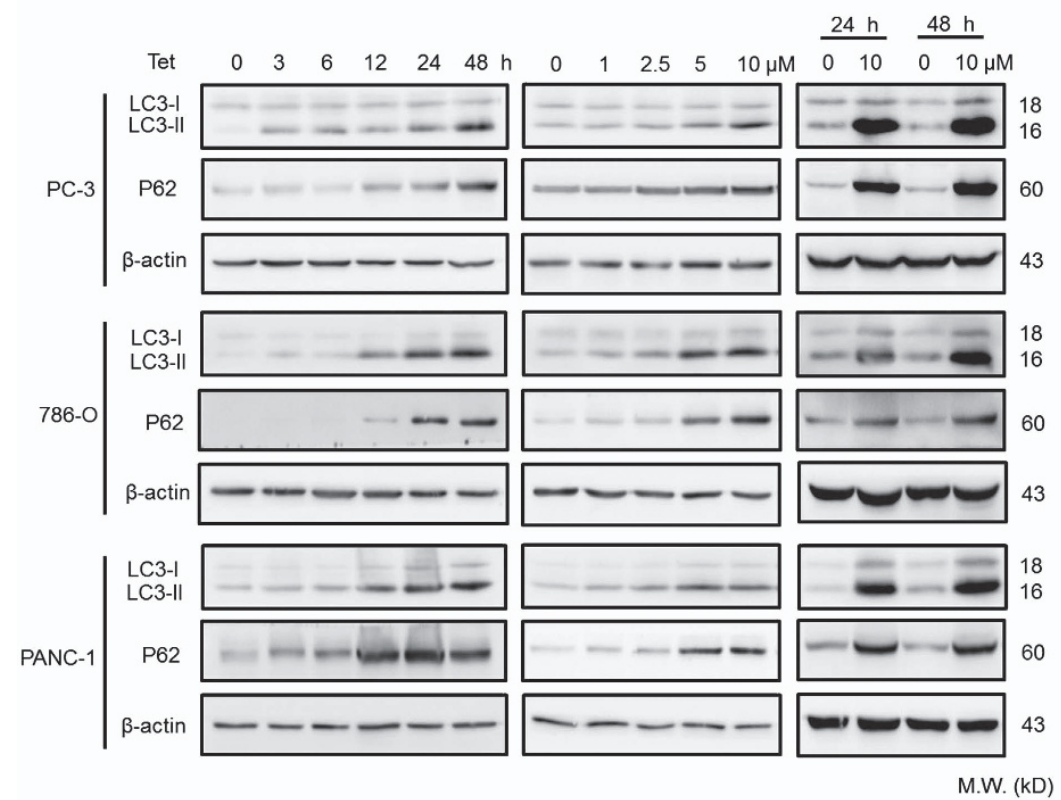

Figure 3 Tet induces autophagosome accumulation in vitro and in vivo. (a) Transmission electron microscopy of PC-3 and 786-0 cells in the presence or absence of Tet $(10 \mu \mathrm{M}, 6 \mathrm{~h})$. Prostate cancer xenografts with or without Tet treatment are shown. Autophagic vacuoles (black arrows) accumulated in the tumor cells. (b) Both PC-3 and 786-O cells were transfected with the EGFP-LC3 plasmid. After $24 \mathrm{~h}$, the cells were incubated with Tet (10 $\mu \mathrm{M}$ ) or vehicle (Ctrl) for $6 \mathrm{~h}$ and visualized with confocal microscopy (upper panel; scale bars, $20 \mu \mathrm{m}$ ). The number of punctate EGFP-LC3 in each cell was counted, and at least 100 cells were included for each group (lower panel). (c) The lipidation of LC3 and the levels of SQSTM1/p62 in PC-3, 786-O, and PANC-1 cells were detected by western blotting in cells treated with Tet as indicated. (d) Primary prostate and renal cancer cells were incubated with Tet $(10 \mu \mathrm{M})$ or vehicle (Ctrl) for $6 \mathrm{~h}$ and the expression of LC3 and SQSTM1/p62 was then measured by westem blotting. (e-g) Autophagy was detected in prostate cancer xenografts by analyzing both the levels of LC3-II and SQSTM1/p62 (e) in mice injected intraperitoneally with Tet or vehicle (Ctrl). The relative intensities of LC3-II (f) and SQSTM1/p62 (g) are shown; $n=5$ for each group, representative graphs are shown; ${ }^{*} P<0.05$, ${ }^{*} P<0.01$ versus Ctrl group

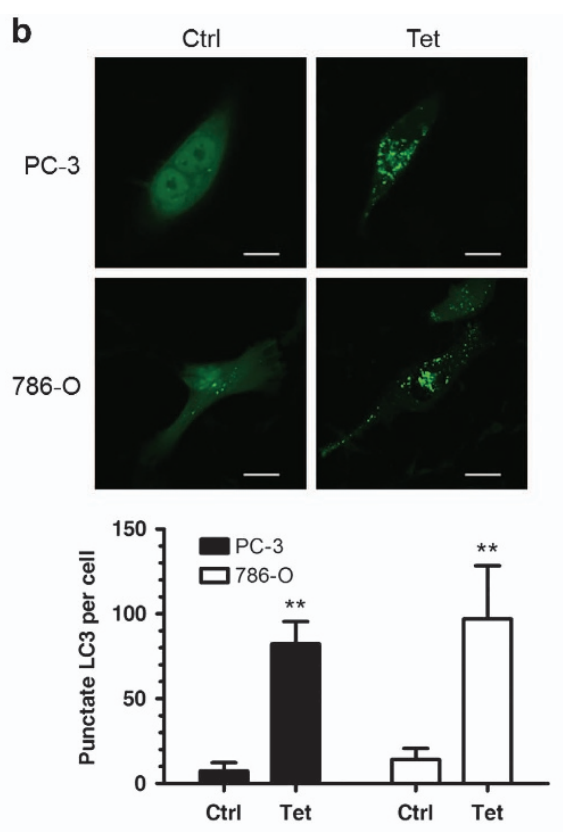

d
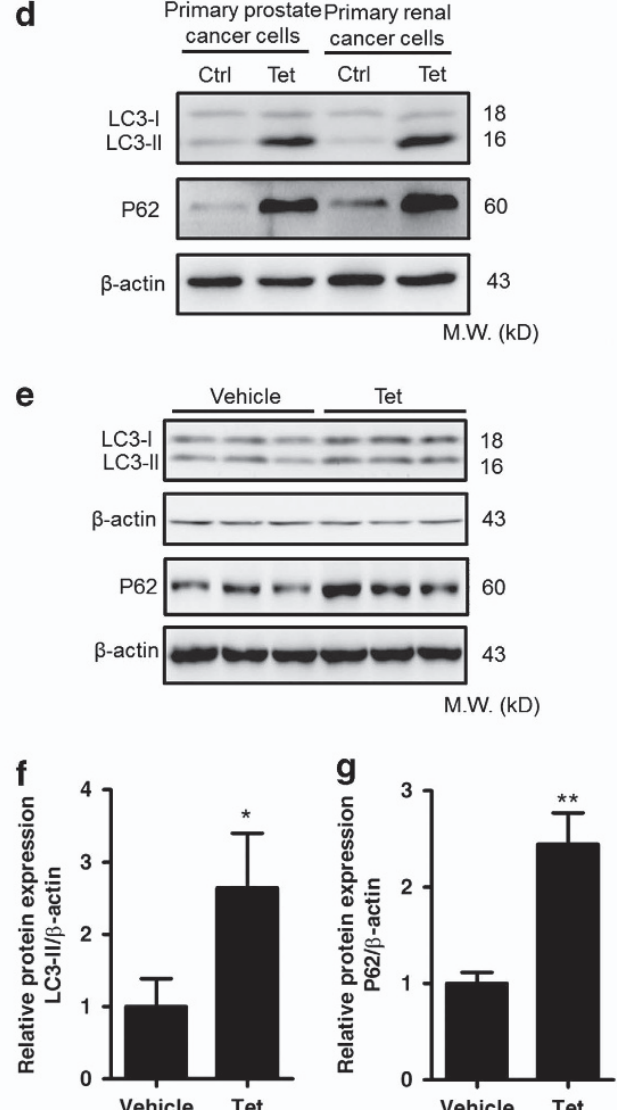
a
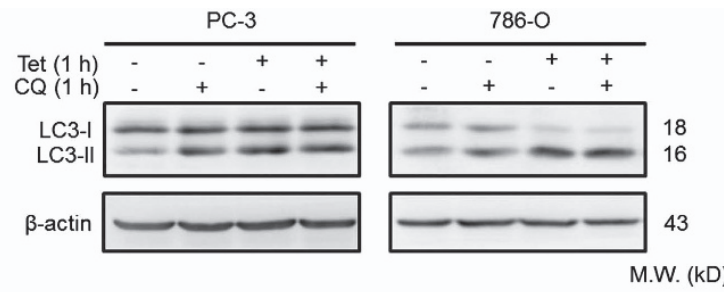

b
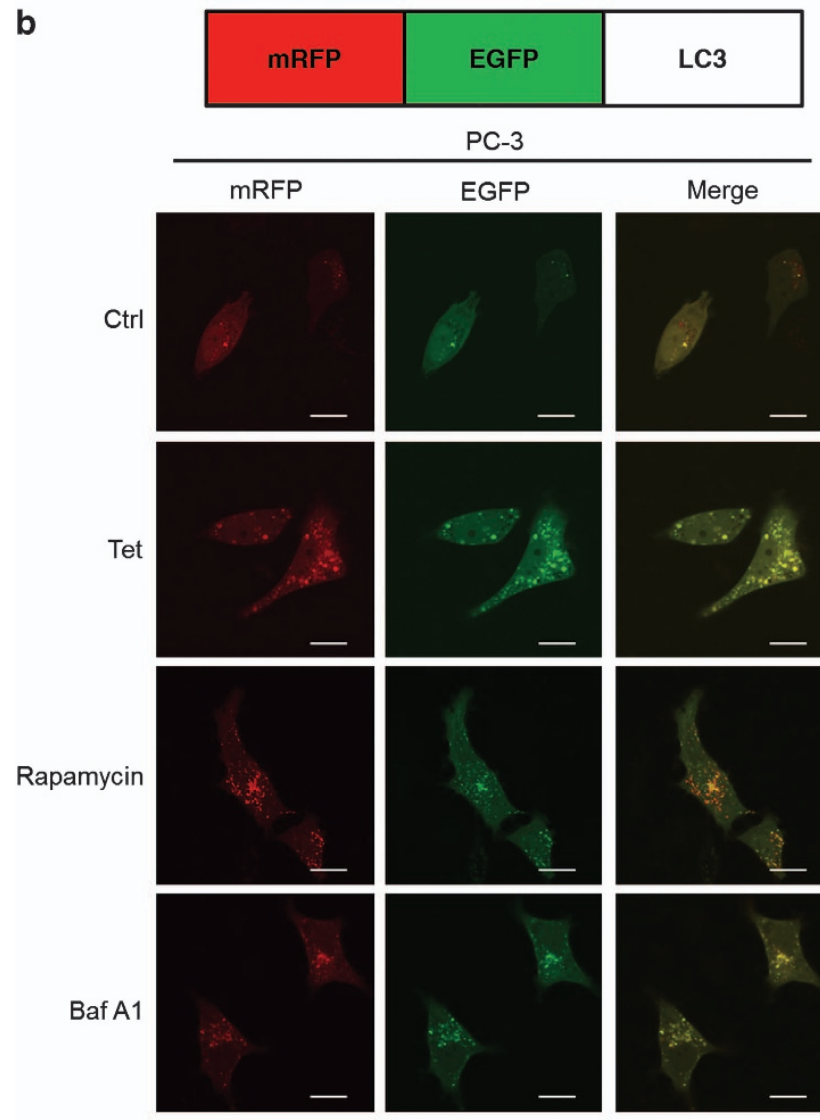

C

EGFP puncta per cell

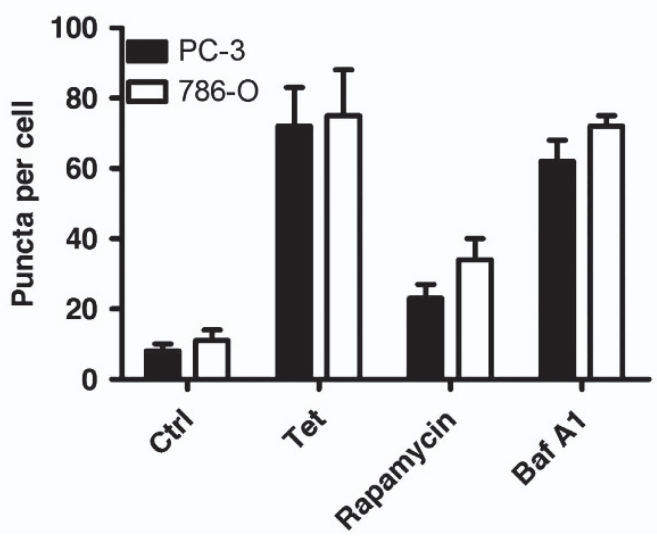

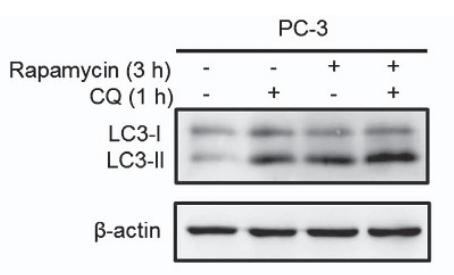

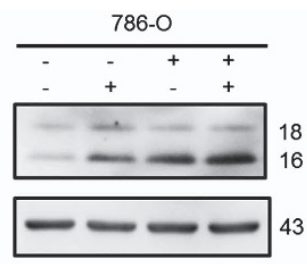

M.W. (kD)

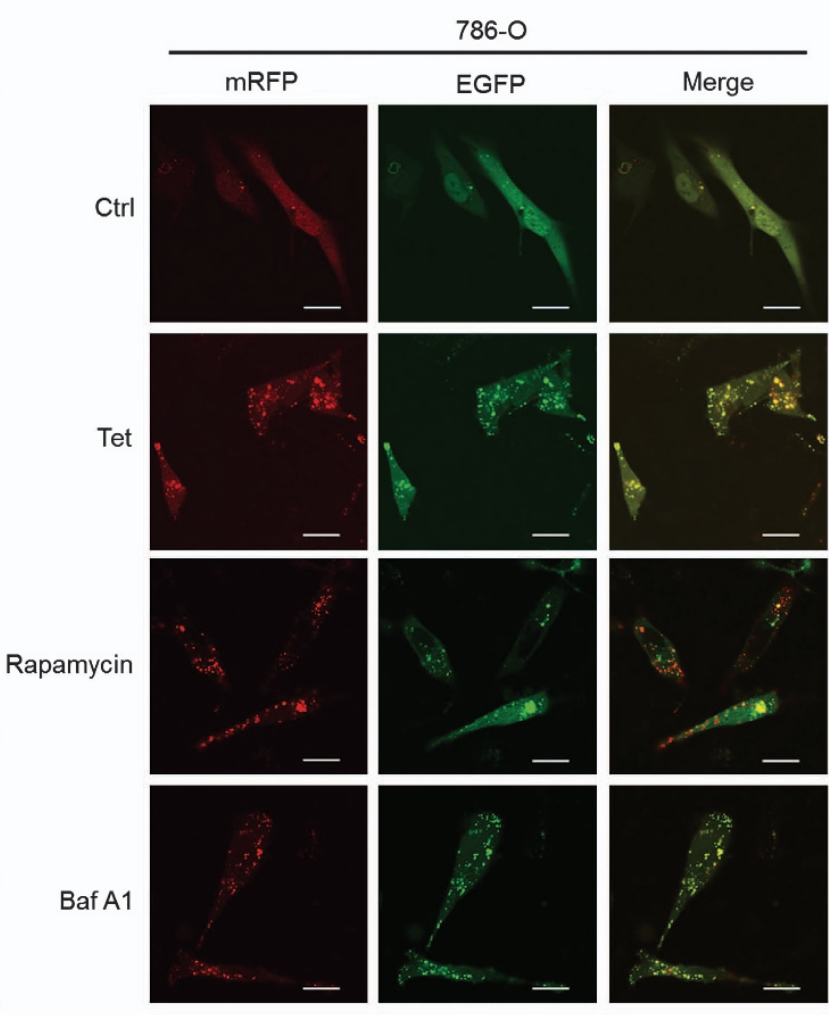

Autolysosomes per cell (mRFP-EGFP puncta)

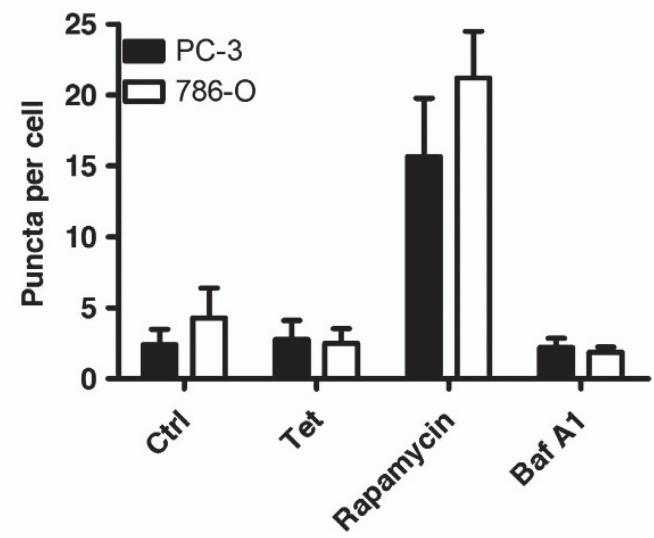

Figure 4 Tet blocks autophagic flux in the degradation stage. (a) LC3 turnover assays were performed in the presence or absence of $10 \mu \mathrm{M} C \mathrm{C}$ in cells treated with Tet $(10 \mu \mathrm{M}$; left) or rapamycin (200 nM; right) for the indicated time, followed by western blotting to detect LC3 in both PC-3 and 786-O cells. (b) Schematic diagram of the tfLC3 plasmid (upper panel). PC-3 cell (left) and 786-O cells (right) expressing tfLC3 were incubated with Tet (10 $\mu \mathrm{M}$ ) for $3 \mathrm{~h}$, rapamycin (200 nM) for $3 \mathrm{~h}$, or bafilomycin A1 (Baf A1) $(200 \mathrm{nM})$ for $1 \mathrm{~h}$. Representative fluorescent images are shown (scale bars, $20 \mu \mathrm{m}$ ). (c) Autophagosomes per cell (number of EGFP puncta per cell; left panel) and autolysosomes per cell (number of mRFP puncta per cell - number of EGFP per cell; right panel) were quantified, and at least 100 cells were included for each group 
be affected as well. We therefore followed the change in Iysosomal acidity upon Tet treatment by using LysoSensor Yellow/Blue DND-160 to measure pH changes in lysosomes. As previously described, ${ }^{22}$ the lysosensor produces blue fluorescence (excitation/emission wavelengths: $329 / 440 \mathrm{~nm}$ ) in neutral environments, but changes to yellow fluorescence (excitation/emission wavelengths: $384 / 540 \mathrm{~nm}$ ) in more acidic environments. Cells treated with bafilomycin A1 $(400 \mathrm{nM})$ for $1 \mathrm{~h}$ were used as a positive control of an increase in lysosomal $\mathrm{pH}$. Our results showed that Tet markedly elevated $\mathrm{pH}$ in a concentration-dependent manner (Figures $5 a-c)$. Fluorescence microscopy of Tet-treated cells examined using the LysoSensor DND-160 probe revealed less green fluorescence (Figure $5 \mathrm{~d}$ ). Taken together, our results indicate that Tet is a potent lysosomal deacidification agent and is accordingly able to block autophagic flux.

Blunting autophagosome formation fails to protect cells against Tet-induced cell death. Tet was reported to induce reactive oxygen species (ROS) release. ${ }^{14}$ Consistently, we observed that the ROS levels were indeed increased in
Tet-treated PC-3 and BEL-7402 cells (Figure 6a). As ROS release could induce autophagy, ${ }^{23}$ we next sought to inhibit the autophagic sequestration with Vps34 inhibitor 3-MA. Vps34 is a required protein for the induction of autophagy, whereas 3-MA blocks this protein to reduce the formation of autophagosomes. $^{24}$ Our results showed that 3-MA (2 mM) treatment was sufficient to inhibit autophagy (Supplementary Figure 6A). If Tet-induced cell death is due to the induction of autophagy, 3-MA treatment could be protective. As shown in Figures $6 \mathrm{~b}$ and $\mathrm{c}$, in Tet-treated PC-3 and 786-O cells, apoptosis was slightly exacerbated by addition of 3-MA. We then blunted autophagy genetic knockdown of Beclin-1 with two different siRNAs to inhibit autophagosome formation (Figure 6d), and similar results were observed (Figures $6 e$ and f).

Treating with Tet in combination of 3-MA or genetic knockdown of Beclin-1 leads to a more complete dual inhibition of both the induction stage and the degradation stage in autophagy. Because the autophagic flux has been already strongly blocked by Tet in the degradation stage and caused a considerable cell death, so cell death was only

a

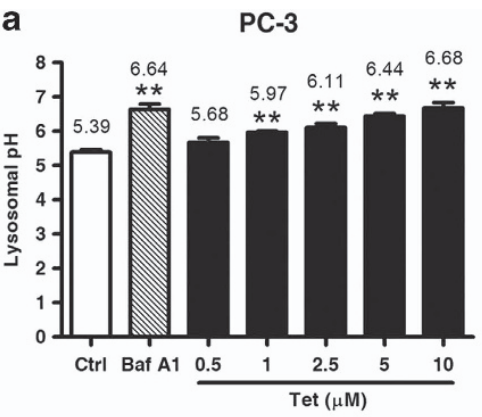

d

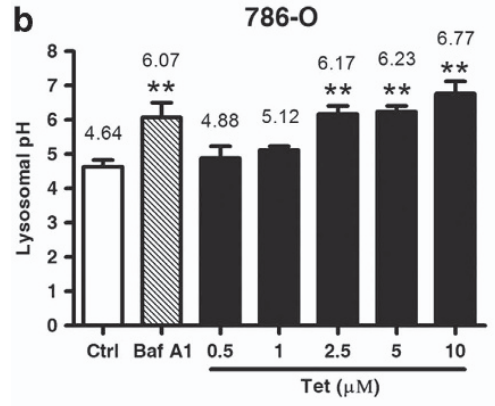

C

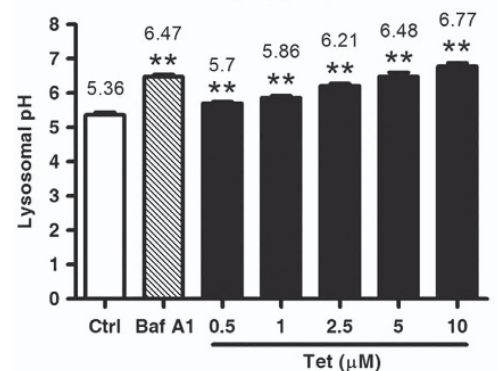

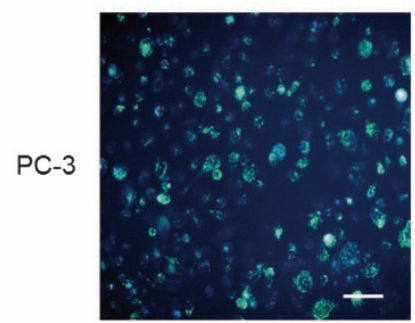

Ctrl

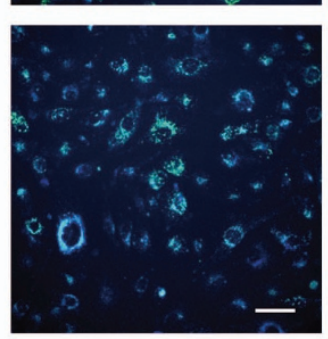

PANC-1

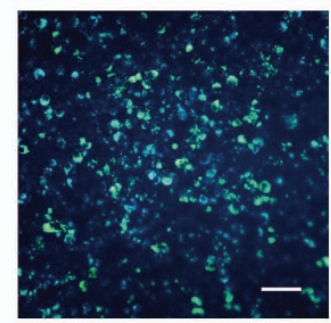

Tet
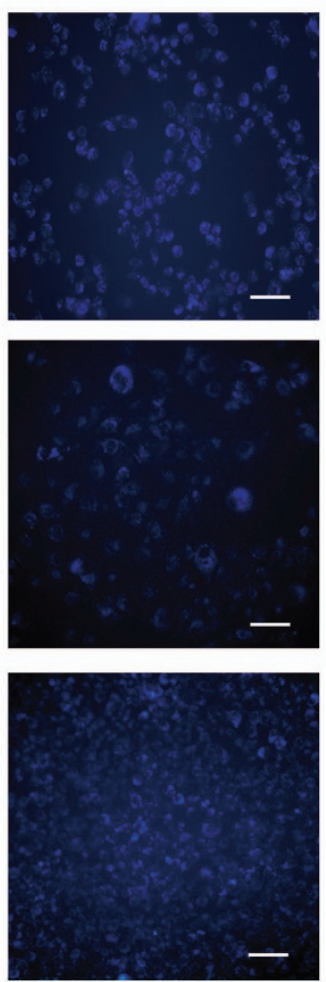

Baf A1
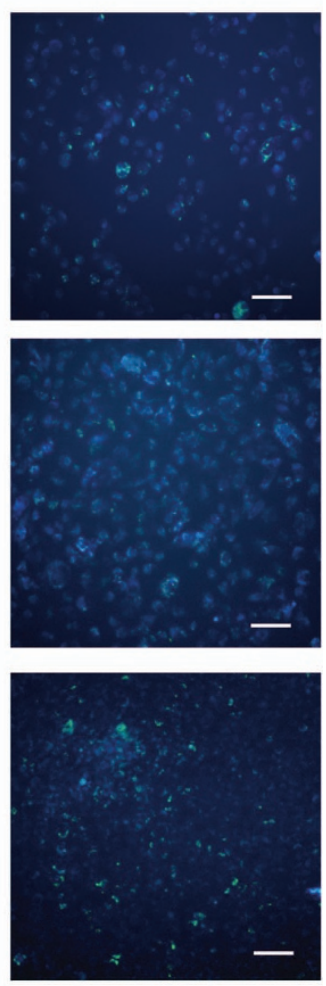

Figure 5 Tet prevents lysosomal acidification in tumor cells. (a-c) PC-3 (a), 786-O (b), and PANC-1 cells (c) were incubated with Tet for the indicated concentration (1 $\mathrm{h}$ ) or, as a positive control, with $400 \mathrm{nM}$ bafilomycin A1 (Baf A1) for $1 \mathrm{~h}$. The pH values in each group are shown. Bars, S.D.; columns, mean, $n=6$; * $P<0.01$ versus the control groups, and (d) cells in each group were examined by fluorescence microscopy using DND-160 lysosensors (scale bars, $25 \mu \mathrm{m}$ ); representative graphs are shown 
a
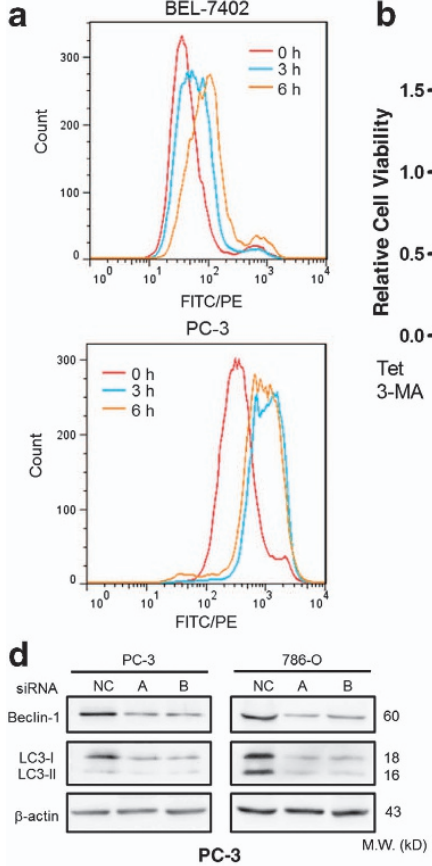

e
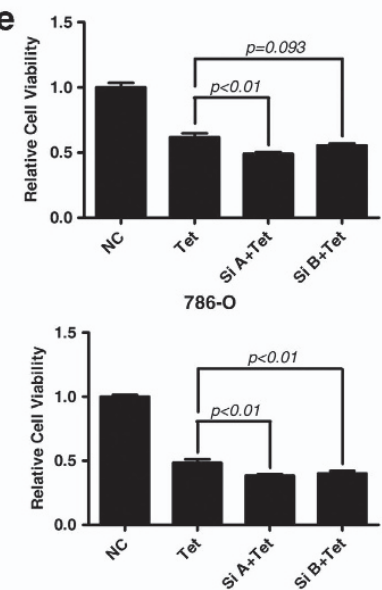

b
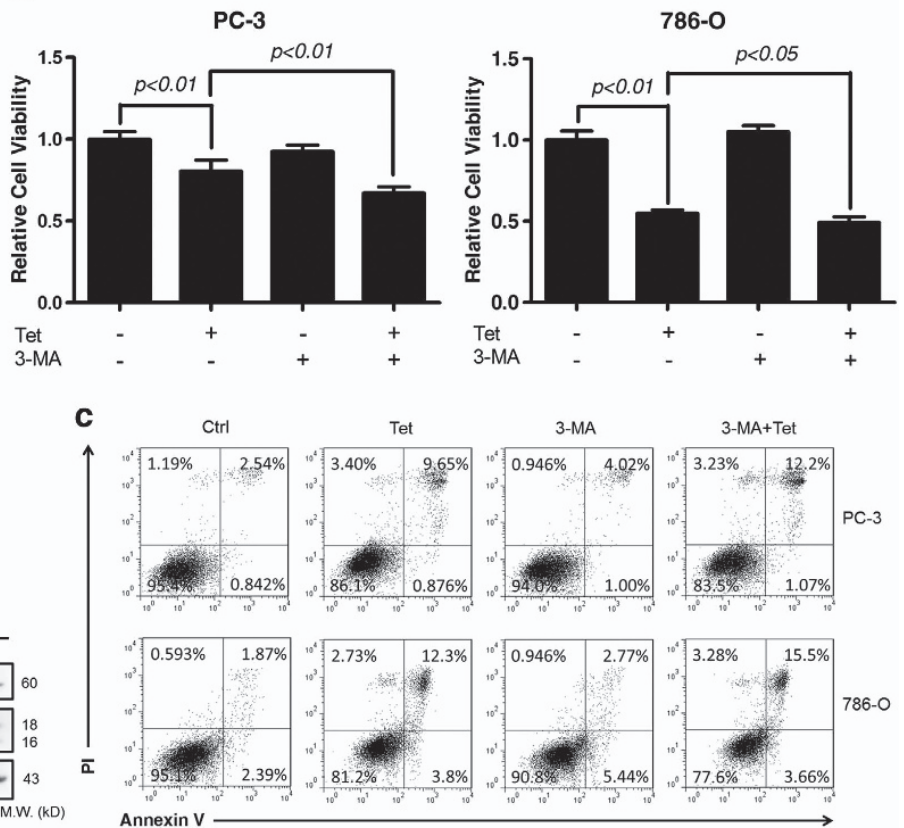

f
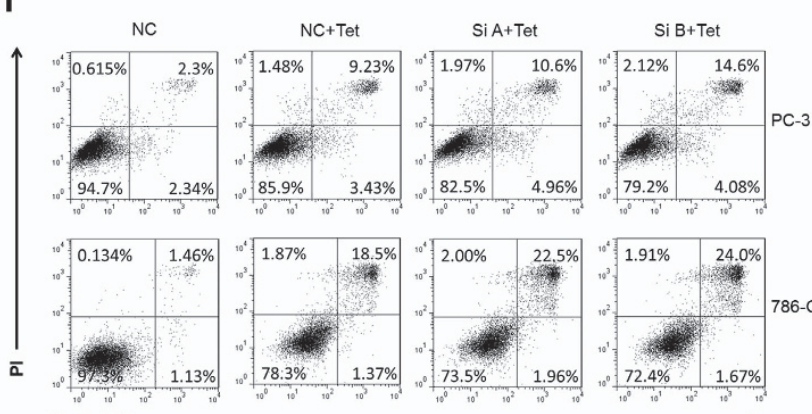

Annexin V

Figure 6 Inhibition of autophagosome formation fails to protect cells from Tet-induced cell death. (a) Both BEL-7402 and PC-3 cells were pre-incubated with Tet (10 $\mu \mathrm{M}$ ) for the indicated time and subsequently subjected to DCF fluorescence analysis to evaluate the intracellular ROS. (b) PC-3 and 786-0 cells were pre-incubated with 3-MA $(2 \mathrm{mM})$, an inhibitor of autophagosome formation, for $1 \mathrm{~h}$ after which Tet $(10 \mu \mathrm{M})$ was added or not and the cells were incubated for an additional $24 \mathrm{~h}$. Cell viability was analyzed in CCK-8 assays. The data were obtained from three independent experiments and statistically analyzed using Student's t-test as indicated. (c) Flow cytometry analysis of cells stained with Annexin V/PI to investigate apoptosis under the same conditions as in (b). (d-f) Autophagosome formation was blunted by genetic knockdown of Beclin-1 with two independent siRNAs (d). Cell viability (e) and apoptosis (f) were detected as described as in (b) and (c), respectively. The data were obtained from three independent experiments and statistically analyzed using Student's t-test between groups as indicated

slightly exacerbated in Tet-treated cells by inhibiting autophagosome formation. More importantly, these data provide evidence that Tet-induced cell death is not an 'autophagic cell death'.

Tet induces apoptosis by energy depletion. Blockade of autophagic flux decreases the recycling of cellular fuels, which eventually leads to an insufficient energy supply and thus to cell death. ${ }^{25,26}$ To follow this sequence of events, we measured the intracellular production of ATP in cells incubated with Tet. Indeed, intracellular ATP levels were significantly reduced in PC-3 cells treated with Tet for $12 \mathrm{~h}$ and in 786-O cells for $6 \mathrm{~h}$ (Figure 7a). Phosphorylation of the energy sensor AMPK $\alpha$ on residue T172 was also remarkably increased at $12 \mathrm{~h}$, both in PC-3 and 786-O cells (Figure 7b), indicating a decrease in the cellular production of ATP after incubation of the cells with Tet. In addition, the activation of AMPK $\alpha$ is adaptive, whereas inhibition of its activation with Compound C $(10 \mu \mathrm{M})$ led to decreased viability in Tet-treated cells (Figure 7c; Supplementary Figure 7). As shown in Figure $7 \mathrm{~d}$, oxygen consumption rate (OCR) decreased upon Tet treatment for $6 \mathrm{~h}$, consistent with an impairment of OXPHOS and thus with a direct decrease in ATP production.

The effects of Tet on OXPHOS might have been related to a drop in mitochondrial $\Delta \Psi m$, as shown in Figure 1e. However, the decrease in $\Delta \Psi m$ would also seem to be an early event 
followed by the induction of apoptosis. Alternatively, the decrease in OXPHOS might reflect an insufficiency of TCA carbon substrates, for the absence of autophagy-supplied substrates may also cause mitochondrial dysfunction. ${ }^{27}$ In pancreatic cancer cells, blocking autophagy flux with $\mathrm{CQ}$ increases the glucose uptake to compensate energy depletion. ${ }^{26}$ In Tet-treated cells, we observed the glucose uptake was marked repressed by an unknown mechanism, which further exacerbated energy depletion upon autophagy inhibition (Figure 7e). We then incubated PC-3 and 786-O cells with Tet and $10 \mathrm{mM}$ methyl pyruvate (MP), as a source of substrates for the TCA cycle. As expected, excess MP partially restored ATP production, accompanied by a decrease in the phosphorylation of AMPK $\alpha$ at T172 (Figures $7 f$ and $g$ ). Thus, despite Tet stimulation, both PC-3 and 786-O cells were partially rescued when provided with an excess of TCA cycle substrates, as illustrated by the markedly decreased rate of apoptosis in MP + Tet-treated cells compared with cells treated with Tet alone (16.75\% versus $9.41 \%$ in PC-3 cells and $18.11 \%$ versus $8.02 \%$ in $786-\mathrm{O}$ cells) (Figure 7h). Consistent with these results, the levels of cleaved caspase-3 and cleaved PARP were diminished in MP + Tet-treated cells compared with cells treated only with Tet (Figure 7i). Interestingly, in the former, $\Delta \Psi \mathrm{m}$ also increased (Figure 7j), supporting that the Tet-related decrease in OXPHOS is caused (at least partially) by a reduction in the supply of TCA substrates. These data suggest a sequence of events in which Tet inhibits autophagic flux and thereby reduces the supply of TCA substrates, leading to energy insufficiency and thus to apoptosis.

\section{Discussion}

Little is known about the ability of Tet, a traditional Chinese medicine, to affect autophagy in a wide range of cell types. In this study, we show that Tet is a potent lysosomal alkalizing agent that exhibits broad antitumor activity in various human tumor cell lines. In contrast with previous studies, we elucidate that Tet actually blocks rather than activates autophagic flux in tumor cells, mainly by neutralizing lysosomal acidity. Besides, we established the relationship among autophagy inhibition, metabolism, and apoptosis in Tet-treated cells.

Autophagy serves as a catabolic pathway to maintain the cellular energy supply and homeostasis. The cargo is degraded by lysosomal hydrolytic enzymes and the amino acids, fatty acids, sugars, and nucleosides thus released are recycled. ${ }^{27,28}$ Only assessing the amount of autophagosome or LC3-II level is insufficient to reflex the entire process of autophagy under certain treatment. Gong et al. ${ }^{14}$ previously reported that Tet induces autophagy in HCC cell lines. However, they did not investigate the effects of Tet on autophagy of the later stage. In contrast, our data provide strong evidence that the autophagic flux is actually blocked by Tet in the degradation stage, which enriches the understanding of its effects on autophagy.

In order to investigate whether Tet is able to induce autophagosome formation as described, ${ }^{14}$ we tried to decrease the concentrations of Tet to weaken its effects on lysosome. But we failed to observe a significant activation of autophagy in any tested concentrations indicated in Figure 3c.
The increase of LC3-II was constitutively accompanied with an increase of SQSTM1/p62 (Figure 3c), which indicates the blockade of autophagy. LC3 turnover assays also confirmed the autophagic flux was indeed blocked by Tet of any of the tested concentrations (data not shown). These results support the conclusion that the inhibition of autophagy has a central role in Tet-mediated autophagosome accumulation. However, the increased levels of ROS induced by Tet or the subsequent activation of AMPK after $12 \mathrm{~h}$ might further increase the number of autophagosomes, as adaptive responses to cope with oxidative or energy depletion stresses.

In autophagy, the lysosome is the only way to degrade cargos, so complete inhibition of the lysosome always causes serious impairment of autophagic flux. Low $\mathrm{pH}$ is required for the hydrolases in the lysosome. ${ }^{17}$ Our results suggest that Tet severely suppresses lysosomal acidification, thus preventing the activation of lysosomal enzymes and thereby their ability to degrade the cargo of the autolysosome, which in turn leads to a blockade of autophagic flux. CQ and its derivatives are widely used lysosomal alkalizing agents. ${ }^{29}$ On the basis of the upregulation of autophagy in cancer cells treated with chemotherapeutic agents, autophagy inhibition has become a promising strategy for cancer treatment. Both $\mathrm{CQ}$ and hydroxychloroquine are currently being evaluated in clinical trials aimed at assessing their efficacy.

Until recently, the detailed relationship between autophagy and cell death is still not very clear. In our research, the so-called 'autophagic cell death' is excluded, because inhibiting autophagy with 3-MA or knockdown of Beclin-1 failed to rescue Tet-treated cells. In Tet-induced cell death, a significant late-stage of apoptosis was observed, and MP treatment could protect against this kind of cell death. We speculate that the high levels of late-stage apoptosis might be due to sharply decreased energetic metabolism after $12 \mathrm{~h}$. Rapid cell growth and proliferation demand a large quantity of energy, which in cancer cells is met by their ability to adaptively reprogram their metabolism, including the upregulation of aerobic glycolysis and glutaminolysis. ${ }^{30}$ Autophagy is crucial to support cancer cell growth and metabolism, by providing substrates for both biosynthesis and energy generation. Our observations that Tet reduces cellular ATP production and that the provision of additional carbon substrates protects tumor cells from apoptosis support a relationship between Tet-induced apoptosis and energy depletion during the drug-induced blockade of autophagic flux. Our results demonstrate that Tet could induce significant phosphorylation of AMPK. During energetic stresses, the activation of AMPK limits protein synthesis and cell growth to protect against apoptosis through the TSC2/mTOR pathway. ${ }^{31}$ Therefore, inhibition of AMPK activation exacerbates Tet-induced apoptosis. These findings indicate that energetic impairment is required in Tet-induced apoptosis.

In conclusion, our study showed that Tet is a potent lysosomal alkalizing agent that impairs the lysosomal function of cancer cells and causes a blockade of the autophagic flux, leading to energy depletion and thus apoptosis. Tet, as a traditional Chinese medicine, has long been widely used in clinical practice in China. Our research recommends the evaluation of Tet as a potential cancer chemopreventive and chemotherapeutic drug, based on its demonstrated ability to inhibit autophagy. 
Materials and Methods

Chemicals and reagents. Tet (sc-201492; Santa Cruz Biotechnology, Dallas, TX, USA) was dissolved in a drop of $0.1 \mathrm{mM} \mathrm{HCl}$, then neutralized with $\mathrm{NaHCO}_{3}$ to $\mathrm{pH} 7.4$, and diluted with PBS to achieve a concentration of $10 \mathrm{mM}$ for storage. For in vivo studies, Tet was suspended in $0.9 \%(\mathrm{w} / \mathrm{v}) \mathrm{NaCl}$ to $12 \mathrm{~g} / \mathrm{l}$. CQ (C6628), bafilomycin A1 (B1793), and compound C (P5499) were purchased from Sigma-Aldrich (St. Louis, MO, USA). 3-MA (sc-205596), anti- $\beta$-actin (sc-47778), anti-Beclin-1 (sc-11427), and Necrostatin-1 (sc-200142) were purchased from Santa Cruz Biotechnology. Z-VAD-FMK (ALX-260-020) was purchased from Enzo Life Sciences (New York, NY, USA). MP (127650250) was obtained from Acros (Geel, Belgium). The antibody against LC3B (L7543) was purchased from Sigma-Aldrich. The following reagents and antibodies were from a

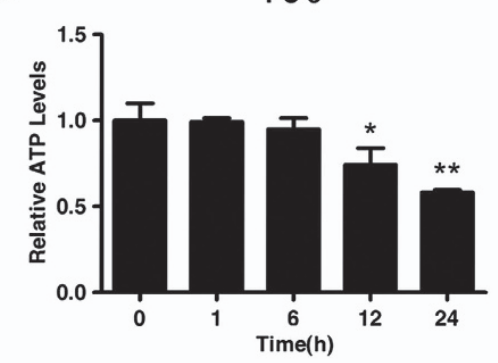

$786-0$

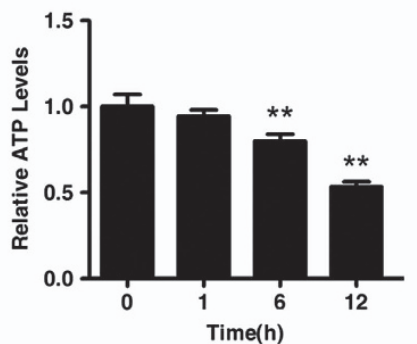

b

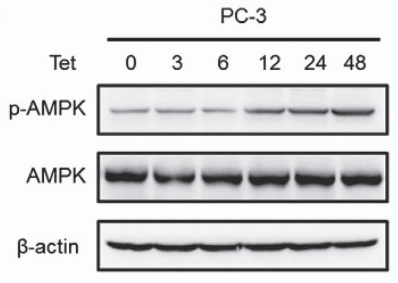

C

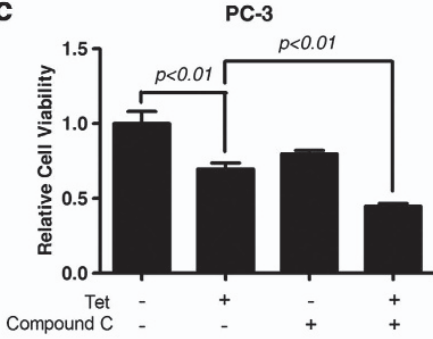

g

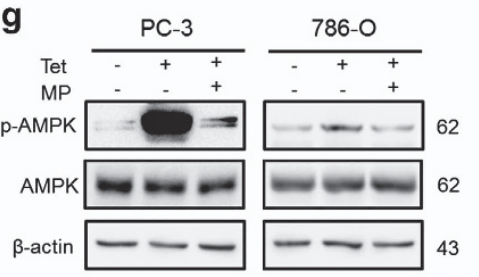

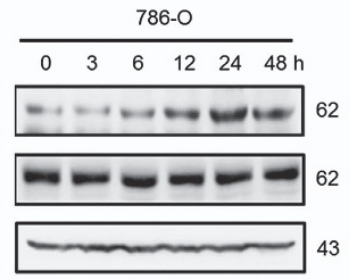

M.W. (kD)

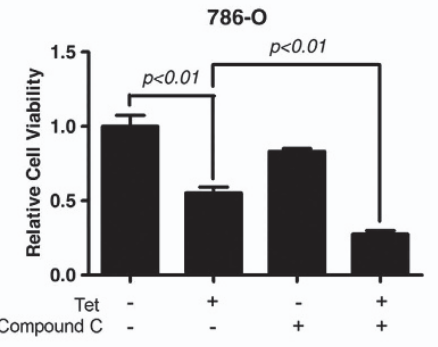

i

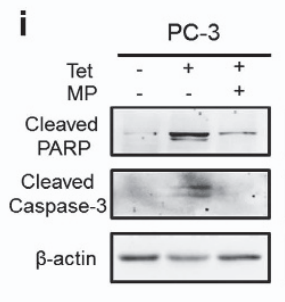

$(\mathrm{kD})$ d $\quad$ PC-3
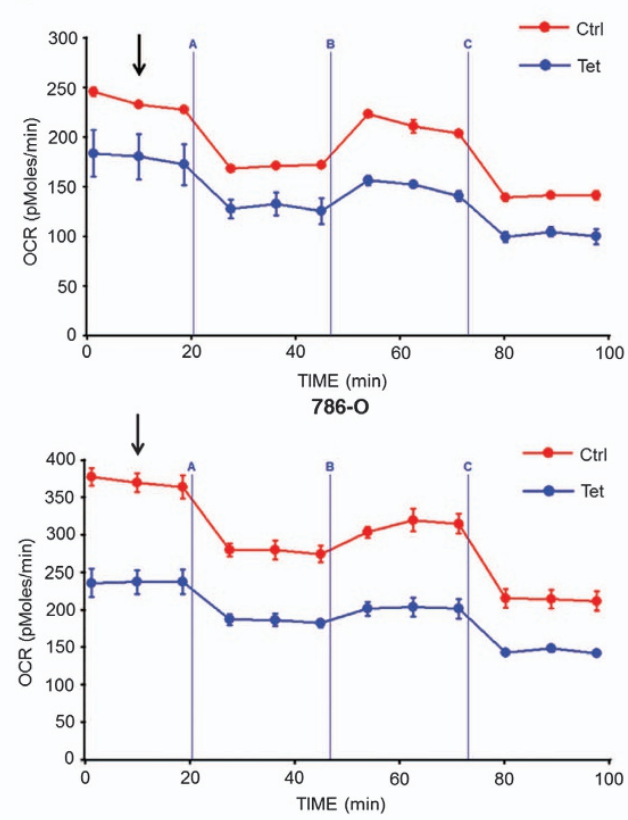

f
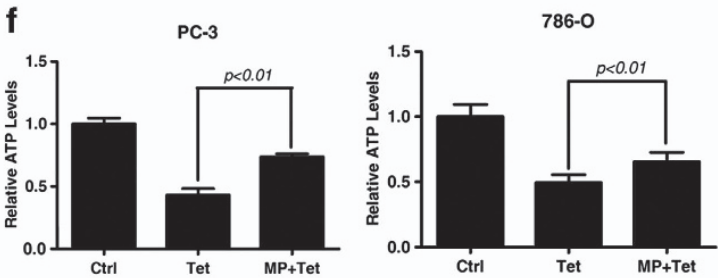

h

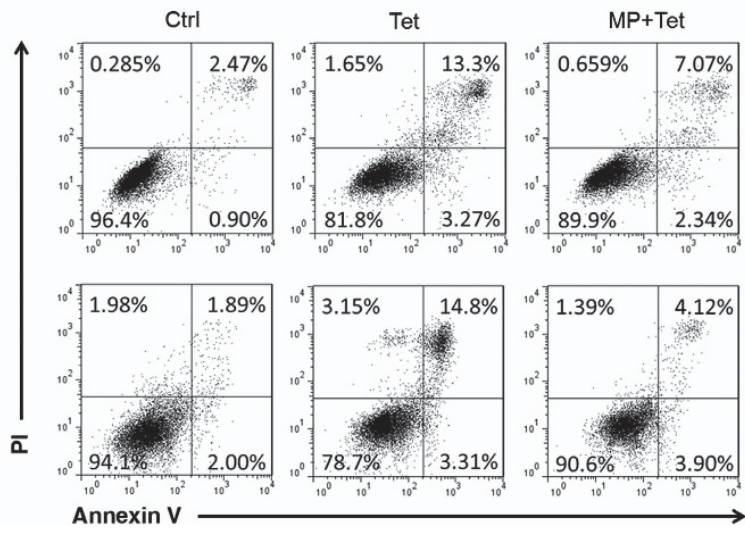

PC-3

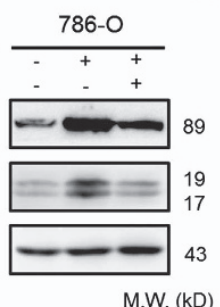

M.W. (kD)
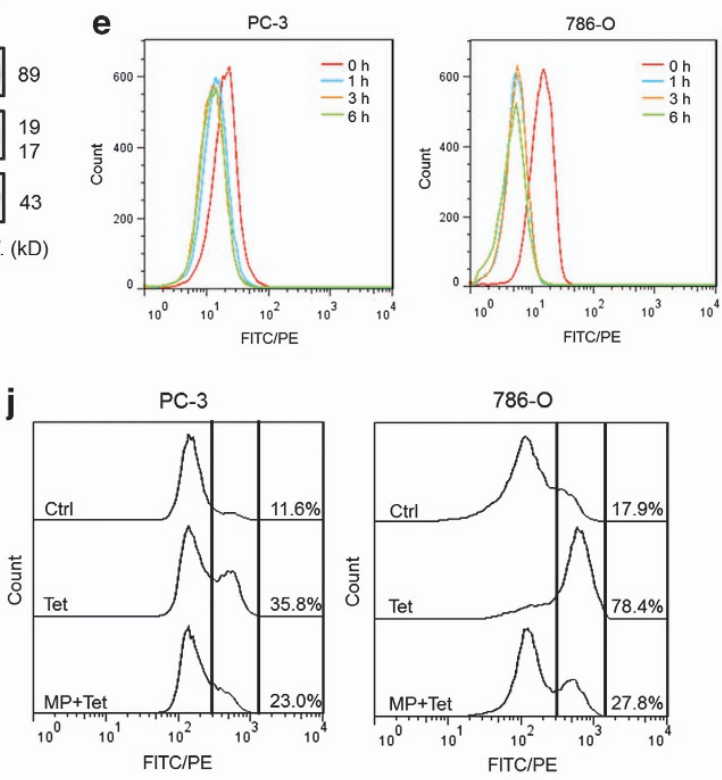

$786-0$ 
Cell Signaling Technology (Boston, MA, USA): rapamycin (9904), cleaved caspase-3 (9664), cleaved PARP (5625), SQSTM1/p62 (5114), p-AMPK $\alpha$ (T172) (2535), and AMPK $\alpha$ (2532). Lipofectamine 2000 (Invitrogen, San Diego, CA, USA; 11668) was used to transfect cells with plasmids or siRNA.

Culture of tumor cell lines. The prostatic cancer cell line PC-3, the renal carcinoma cell line 786-O, the bladder cancer cell line EJ and J82, the hepatoma cell line BEL-7402 were obtained from the National Platform of Experimental Cell Resources for Sci-Tech (Beijing, China). The other cell lines used in this study were purchased from the American Type Culture Collection (Rockville, MD, USA). PC-3, 786-0, EJ, BEL-7402, HeLa (cervical cancer), NCl-H460 (lung cancer), HepG2 (hepatoma), and DU145 (prostatic cancer) cells were maintained in RPMI-1640 medium (Hyclone, Logan, UT, USA; SH30027.01). PANC-1 (pancreatic cancer) cells and MCF-7 (breast cancer) cells were cultured in Dulbecco's modified Eagle's medium (Hyclone, SH30022.01B). J82 cells were grown in minimum essential medium (Hyclone, SH30024.01B). All media contained $10 \%$ fetal bovine serum (Biological Industries, Beit-Haemek, Israel; 04-001-1). Stable EGFP-LC3-transformed HeLa cells were obtained as a gift from Dr. Yongqing Shen (Hebei Medical University, Hebei, China). All cultures were incubated at $37^{\circ} \mathrm{C}$ in a humidified atmosphere containing $5 \% \mathrm{CO}_{2}$

Isolation and culture of primary tumor cells. Tumor samples obtained at the time of tumor resection were washed with cold PBS, cut into small pieces, and digested with collagenase to obtain single-cell suspensions. These cells were washed twice with PBS, resuspended in medium, and cultured as described above. Primary prostate cancer cells and primary renal cancer cells were cultured in a prostate epithelial cell medium (Sciencell, Carlsbad, CA, USA; 4411) and DMEM/F12 (Hyclone, SH30023.01), respectively. The protocol for the primary tumor cell cultures was approved by the Medical Ethics Committee of Peking University First Hospital.

Animal experiments. Five-week-old male BALB/c nude mice were subcutaneously injected in the right flank with $3 \times 10^{6}$ PC- 3 cells. After 5 days, the mice were randomly distributed into vehicle and treatment groups ( $n=5$ each) and then treated accordingly. Tet was administered via an intraperitoneal injection at doses of $60 \mathrm{mg} / \mathrm{kg}$ every 2 days. The vehicle group received $0.9 \% \mathrm{NaCl}$ only. Vernier calipers were used to measure tumor size every other day after the start of treatment. Tumor volume was calculated using the formula: (length $\times$ width $\left.^{2}\right) / 2$. The protocol for the animal experiment was approved by the Peking University Institutional Animal Care and Use Committee.

Plasmids and RNAi. The EGFP-LC3 plasmid and the mRFP-EGFP-tfLC3 plasmid were kindly provided by Dr. Tamotsu Yoshimori (Osaka University, Osaka, Japan). Beclin-1 and negative control siRNAs were synthesized by GenePharma (Shanghai, China): negative control siRNA, UUCUCCGAACGUGUCACGUTT; siA GCUGCCGUUAUACUGUUCUTT; siB, GUGGAAUGGAAUGAGAUUATT.

Cell proliferation assay. The cells were seeded in 96-well plates, cultured overnight as described above, and treated with Tet or as otherwise noted for the specified period. Cell viability was determined using the Cell Counting Kit-8 (CCK-8) (Dojindo, Kumamoto, Japan; CK04).

Assays of mitochondrial membrane potential, ROS, and apoptosis. Mitochondrial membrane potential $(\Delta \Psi \mathrm{m})$ was evaluated using the probe JC-1 (Invitrogen, M34152). Briefly, cells in each group were harvested, washed twice with cold PBS, centrifuged, and then stained with JC-1 $(2 \mu \mathrm{M})$ for 15 min at $37^{\circ} \mathrm{C}$ and analyzed using flow cytometry (BD PharMingen, San Diego, CA, USA).

The levels of ROS were detected using DCFH-DA probe (Sigma, St. Louis, MO, USA; D6883). Briefly, the cells were cultured and treated with $10 \mu \mathrm{M}$ Tet at the indicated time. Then, the cells were harvested, washed with PBS, and incubated with DCFH-DA $(5 \mu \mathrm{M})$ in a serum-free medium at $37^{\circ} \mathrm{C}$ in a $5 \% \mathrm{CO}_{2}$ incubator for 20 min, washed twice with PBS again, and analyzed by flow cytometry.

To measure apoptosis, an FITC Annexin-V apoptosis detection kit (BD PharMingen, 556547) was used according to the manufacturer's instructions. The cells were stained with Annexin V/PI and detected with flow cytometry as described above. All flow cytometry data were analyzed with FlowJO software.

Apoptotic cells in xenografts were identified by TUNEL staining using the In Situ Cell Death detection kit (Roche Molecular Biochemicals, Lewes, UK; 11684817910). The tumor tissue samples were treated according to the manufacturer's directions and examined with a fluorescent microscope (Olympus, Tokyo, Japan; IX71).

Transmission electron microscopy. All cells in each group were washed with PBS and harvested by centrifugation at $4{ }^{\circ} \mathrm{C}$. The cell pellets were fixed with $2 \%$ glutaraldehyde overnight at $4{ }^{\circ} \mathrm{C}$ and post-fixed with $1 \%$ osmium tetroxide for $1 \mathrm{~h}$. For xenografts, the tissues were immediately cut into pieces and the extracted tumors were fixed in glutaraldehyde. These samples were subsequently dehydrated stepwise in an ethanol series and embedded in an Epon-Araldite mixture. The ultrathin sections were examined using an electron microscope (JEOL LTD., Tokyo, Japan; 1230).

Western blotting. Whole-cell lysates were prepared in lysis buffer $(50 \mathrm{mM}$ Tris- $\mathrm{HCl}, \mathrm{pH} 7.4,150 \mathrm{mM} \mathrm{NaCl}, 1 \mathrm{mM}$ EDTA, $1 \% \mathrm{NP}-40$, and $0.1 \%$ sodium dodecyl sulfate), quantified, and loaded onto SDS-PAGE. After electrophoresis, proteins in the gel were transferred to a nitrocellulose membrane and incubated with primary antibodies at $4{ }^{\circ} \mathrm{C}$ overnight. The membrane was incubated with HRP-conjugated secondary antibodies for $1 \mathrm{~h}$ at room temperature and then washed with TBST. The antibody complexes in the immunoblots were detected by chemiluminescence using an HRP substrate (Millipore, Bedford, MA, USA; WBKLS0100) and visualized using a G:BOX Chemi Gel Documentation System (Syngene, Frederick, MD, USA).

Autophagy assays. Autophagy was assessed in cells transiently transfected with the EGFP-LC3 plasmid and incubated for $24 \mathrm{~h}$. The transfected cells were observed using fluorescence microscopy (Olympus, IX71), acquiring the images by confocal microscopy (Olympus, FV1000). The number of punctate EGFP-LC3 per cell was counted, and at least 100 cells were included for each group. To determine the formation of autophagosomes and autolysosomes, tfLC3 plasmid was transfected into PC-3 and 786-0 cells using Lipofectamine 2000 and incubated for $24 \mathrm{~h}$. Images were acquired using confocal microscopy. The number of autophagosomes (number of EGFP puncta per cell) and autolysosomes (number of mRFP puncta per cell minus number of EGFP puncta per cell) were quantificated per cell, and at least 100 cells were included.

Lysosomal studies. Lysosomal $\mathrm{pH}$ measurements were performed as described previously. ${ }^{32-35}$ In brief, the cells were cultured in a black-walled, clear-bottomed 96-well plate (Corning Inc., New York, NY, USA) and grown to

Figure 7 Tet induces apoptosis by energy depletion. (a) Intracellular ATP production in PC-3 or 786-0 cells treated with Tet $(10 \mu \mathrm{M})$ for the indicated time was measured. Relative ATP levels are expressed as the fold change with respect to the control; $n=6,{ }^{*} P<0.05{ }^{* *} P<0.01$ versus the $0 \mathrm{~h}$ group. (b) $P C-3$ and $786-0$ cells were treated as in (a), after which the phosphorylation of AMPK $\alpha$ at T172, as an energy sensor, was detected by western blotting. (c) PC-3 and 786-O cells were pre-incubated with compound C $(10 \mu \mathrm{M})$, an inhibitor of AMPK $\alpha$, for $1 \mathrm{~h}$ after which Tet $(10 \mu \mathrm{M})$ was added or not and incubated for an additional $24 \mathrm{~h}$. Cell viability was analyzed in CCK-8 assays; $n=6,{ }^{*} P<0.01$ versus the indicated groups. (d) OXPHOS in PC-3 and 786-0 cells treated with Tet $(10 \mu \mathrm{M})$ for $6 \mathrm{~h}$ (blue) was measured as oxygen consumption normalized to protein concentration and compared with nontreated groups (red). Basal mitochondrial respiration (arrow) and ATP-coupled oxygen consumption through OXPHOS (1 $\mu \mathrm{M}$

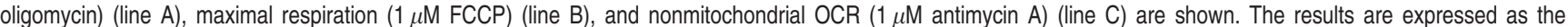
mean \pm S.D. of three independent experiments. (e) Cells were treated with Tet $(10 \mu \mathrm{M})$ for the indicated time; glucose uptake was determined following $2 \mathrm{~h}$ exposure to 2 NBDG. (f) Intracellular ATP levels can be partially restored by the addition of $10 \mathrm{mM} \mathrm{MP.} \mathrm{PC-3} \mathrm{and} \mathrm{786-0} \mathrm{cells} \mathrm{were} \mathrm{treated} \mathrm{with} \mathrm{Tet}(10 \mu \mathrm{M})$ in the presence or absence of MP $(10 \mathrm{mM}$ ) for $24 \mathrm{~h} ; n=6$. (g) In cells treated as in (f), the phosphorylation of AMPK $\alpha$ at T172 was decreased by the addition of MP (10 mM), as detected by western blotting. (h) Apoptosis was analyzed by flow cytometry of cells treated as in (f) for $24 \mathrm{~h}$. The graphs show that MP protects both PC-3 and 786-0 cells from Tet-induced apoptosis. (i) PC-3 and 786-O cells were treated as in (f) for $48 \mathrm{~h}$ and the cleavage of caspase-3 and PARP was then measured by western blotting. Representative results of three independent experiments are shown. A decrease in cleaved caspase-3 and cleaved PARP is seen in the MP + Tet co-treatment groups. (j) $\Delta \Psi m$ was detected by JC-1 staining cells treated as in (f) for $24 \mathrm{~h}$. The panel shows a partial increase in $\Delta \Psi \mathrm{m}$ in the presence of $10 \mathrm{mM} \mathrm{MP}$ 
$100 \%$ confluence. After the indicated treatments, the cells were washed and then incubated with $5 \mu \mathrm{M}$ of LysoSensor Yellow/Blue DND-160 (Invitrogen, L7545) for $5 \mathrm{~min}$. The supernatants were removed, after which $100 \mu \mathrm{l}$ of the appropriate medium was added. Lysosomal $\mathrm{pH}$ values were determined by exposing cells to $10 \mu \mathrm{M} \mathrm{H}^{+} / \mathrm{Na}^{+}$ionophore monensin and $20 \mu \mathrm{M} \mathrm{H}^{+} / \mathrm{K}^{+}$ionophore nigericin in $20 \mathrm{mM}$ MES, $110 \mathrm{mM} \mathrm{KCl}$ and $20 \mathrm{mM} \mathrm{NaCl}$ at $\mathrm{pH} 3.6-6.9$ for $10 \mathrm{~min}$ in each plate. Fluorescence was detected with a Varioskan 96-well plate reader (Thermo Fisher, Waltham, MA, USA). To minimize the 'alkalizing effect' of the probes, all measurements were made $10-15 \mathrm{~min}$ after probe removal. Lysosomal $\mathrm{pH}$ was assessed based on the ratio of light excited at 329 versus $384 \mathrm{~nm}$.

For morphological observations, the cells were incubated with a medium containing the specified drugs for the indicated times, and then stained with $1 \mu \mathrm{M}$ LysoSensor DND-160 for $5 \mathrm{~min}$. After washed with probe-free medium, the samples were viewed using fluorescence microscopy (Olympus, IX71).

Metabolism studies. ATP was measured using the ATPlite luminescence assay system (PerkinElmer, Waltham, MA, USA; 6016943) according to the manufacturer's instructions. The cells were cultured in a white 96-well plate (Greiner Bio-One, Monroe, NC, USA), treated as indicated treatments, and then lysed by the addition of cell lysis solution to each well, with shaking for $5 \mathrm{~min}$. ATP levels were detected by measuring luminescence after the addition of the substrate solution to the cell lysates.

The metabolic profile of cells cultured in a 24-well Seahorse plate and treated with Tet for $6 \mathrm{~h}$ was analyzed using the Seahorse XF24 analyzer (Seahorse Bioscience, Billerica, MA, USA). Basal mitochondrial respiration was measured, after which the following four pharmacologic inhibitors, each at a concentration of $1 \mu \mathrm{M}$, were sequentially injected: oligomycin, a reversible inhibitor of ATP synthase in which ATP-coupled oxygen consumption is mediated by oxidative phosphorylation (OXPHOS); FCCP, a mitochondrial uncoupler that allows a measurement of maximum OXPHOS respiration capacity; rotenone and antimycin $\mathrm{A}$, both of which allow the precise measurement of nonmitochondrial respiration.

For analyzing glucose uptake, cells were incubated with $100 \mu \mathrm{M}$ 2-NBDG (Invitrogen) for $2 \mathrm{~h}$. Fluorescence was measured using flow cytometry.

Statistical analysis. Data were expressed as the mean \pm S.D. of at least three independent experiments. Differences between groups were evaluated by Student's $t$-test or an analysis of variance least significant difference test. Significance was defined as $P<0.05$.

\section{Conflict of Interest}

The authors declare no conflict of interest.

Acknowledgements. We thank Dr. Tamotsu Yoshimori from the Osaka University for the EGFP-LC3 and tfLC3 plasmids, Mr. Yi Ding for assistance in flow cytometric analysis and Dr. Yongqing Shen from the Hebei Medical University for providing the stable EGFP-LC3-transformed HeLa cells.

1. Levine B, Klionsky DJ. Development by self-digestion: molecular mechanisms and biological functions of autophagy. Dev Cell 2004; 6: 463-477.

2. Chen YJ. Potential role of tetrandrine in cancer therapy. Acta Pharmacol Sin 2002; 23: 1102-1106.

3. Lai JH. Immunomodulatory effects and mechanisms of plant alkaloid tetrandrine in autoimmune diseases. Acta Pharmacol Sin 2002; 23: 1093-1101.

4. Koh SB, Ban JY, Lee BY, Seong YH. Protective effects of fangchinoline and tetrandrine on hydrogen peroxide-induced oxidative neuronal cell damage in cultured rat cerebellar granule cells. Planta Med 2003; 69: 506-512.

5. Li SY, Ling LH, Teh BS, Seow WK, Thong YH. Anti-inflammatory and immunosuppressive properties of the bis-benzylisoquinolines: in vitro comparisons of tetrandrine and berbamine. Int J Immunopharmacol 1989; 11: 395-401.

6. Liu QY, Li B, Gang JM, Karpinski E, Pang PK. Tetrandrine, a Ca ++ antagonist: effects and mechanisms of action in vascular smooth muscle cells. J Pharmacol Exp Ther 1995; 273: 32-39.

7. Chen $\mathrm{Y}, \mathrm{Chen} \mathrm{JC}$, Tseng SH. Effects of tetrandrine plus radiation on neuroblastoma cells. Anticancer Res 2009; 29: 3163-3171.

8. Sun X, Xu R, Deng Y, Cheng H, Ma J, Ji J et al. Effects of tetrandrine on apoptosis and radiosensitivity of nasopharyngeal carcinoma cell line CNE. Acta Biochim Biophys Sin (Shanghai) 2007; 39: 869-878.
9. Sun XC, Cheng HY, Deng YX, Shao RG, Ma J. Tetrandrine: a potent abrogator of G2 checkpoint function in tumor cells and its mechanism. Biomed Environ Sci2007; 20: 495-501.

10. Dai CL, Xiong HY, Tang LF, Zhang X, Liang YJ, Zeng MS et al. Tetrandrine achieved plasma concentrations capable of reversing MDR in vitro and had no apparent effect on doxorubicin pharmacokinetics in mice. Cancer Chemother Pharmacol 2007; 60: 741-750.

11. Xu WL, Shen HL, Ao ZF, Chen BA, Xia W, Gao F et al. Combination of tetrandrine as a potential-reversing agent with daunorubicin, etoposide and cytarabine for the treatment of refractory and relapsed acute myelogenous leukemia. Leuk Res 2006; 30: 407-413.

12. Zhu $\mathrm{X}$, Sui $\mathrm{M}$, Fan W. In vitro and in vivo characterizations of tetrandrine on the reversal of P-glycoprotein-mediated drug resistance to paclitaxel. Anticancer Res 2005; 25: 1953-1962.

13. Fu LW, Zhang YM, Liang YJ, Yang XP, Pan QC. The multidrug resistance of tumour cells was reversed by tetrandrine in vitro and in xenografts derived from human breast adenocarcinoma MCF-7/adr cells. Eur J Cancer 2002; 38: 418-426.

14. Gong K, Chen C, Zhan Y, Chen Y, Huang Z, Li W. Autophagy-related gene 7 (ATG7) and reactive oxygen species/extracellular signal-regulated kinase regulate tetrandrine-induced autophagy in human hepatocellular carcinoma. J Biol Chem 2012; 287: 35576-35588.

15. Homewood CA, Warhurst DC, Peters W, Baggaley VC. Lysosomes, pH and the anti-malarial action of chloroquine. Nature 1972; 235: 50-52.

16. Kimura T, Takabatake $Y$, Takahashi A, Isaka $Y$. Chloroquine in cancer therapy: a double-edged sword of autophagy. Cancer Res 2013; 73: 3-7.

17. Settembre C, Fraldi A, Medina DL, Ballabio A. Signals from the lysosome: a control centre for cellular clearance and energy metabolism. Nat Rev Mol Cell Biol 2013; 14: 283-296.

18. Liu C, Gong K, Mao X, Li W. Tetrandrine induces apoptosis by activating reactive oxygen species and repressing Akt activity in human hepatocellular carcinoma. Int J Cancer 2011; 129: 1519-1531.

19. Bjørkøy G, Lamark T, Pankiv S, Øvervatn A, Brech A, Johansen T. Monitoring autophagic degradation of p62/SQSTM1. Methods Enzymol 2009; 452: 181-197.

20. Mizushima N, Yoshimori T, Levine B. Methods in mammalian autophagy research. Cell 2010; 140: 313-326

21. Kimura S, Noda T, Yoshimori T. Dissection of the autophagosome maturation process by a novel reporter protein, tandem fluorescent-tagged LC3. Autophagy 2007; 3: 452-460.

22. Stiernet $P$, Guiot $Y$, Gilon $P$, Henquin JC. Glucose acutely decreases $\mathrm{pH}$ of secretory granules in mouse pancreatic islets. mechanisms and influence on insulin secretion. J Biol Chem 2006; 281: 22142-22151.

23. Scherz-Shouval R, Shvets E, Fass E, Shorer H, Gil L, Elazar Z. Reactive oxygen species are essential for autophagy and specifically regulate the activity of Atg4. EMBO J 2007; 26 . 1749-1760.

24. Wu YT, Tan HL, Shui G, Bauvy C, Huang Q, Wenk MR et al. Dual role of 3-methyladenine in modulation of autophagy via different temporal patterns of inhibition on class I and III phosphoinositide 3-kinase. J Biol Chem 2010; 285: 10850-10861.

25. Yang ZJ, Chee CE, Huang S, Sinicrope FA. The role of autophagy in cancer: therapeutic implications. Mol Cancer Ther 2011; 10: 1533-1541.

26. Yang S, Wang X, Contino G, Liesa M, Sahin E, Ying H et al. Pancreatic cancers require autophagy for tumor growth. Genes Dev 2011; 25: 717-729.

27. Rabinowitz JD, White E. Autophagy and metabolism. Science 2010; 330: 1344-1348.

28. Mizushima N, Komatsu M. Autophagy: renovation of cells and tissues. Cell 2011; 147 : 728-741.

29. Slater AF. Chloroquine: mechanism of drug action and resistance in Plasmodium falciparum. Pharmacol Ther 1993; 57: 203-235.

30. Lozy F, Karantza V. Autophagy and cancer cell metabolism. Semin Cell Dev Biol 2012; 23: 395-401.

31. Inoki K, Zhu T, Guan KL. TSC2 mediates cellular energy response to control cell growth and survival. Cell 2003; 26: 577-590.

32. Baltazar GC, Guha S, Lu W, Lim J, Boesze-Battaglia K, Laties AM et al. Acidic nanoparticles are trafficked to lysosomes and restore an acidic lysosomal $\mathrm{pH}$ and degradative function tocompromised ARPE-19 cells. PLoS One 2012; 7: e49635.

33. Liu J, Lu W, Reigada D, Nguyen J, Laties AM, Mitchell CH et al. Restoration of lysosomal $\mathrm{pH}$ in RPE cells from cultured human and $\mathrm{ABCA} 4(-/-)$ mice: pharmacologic approaches and functional recovery. Invest Ophthalmol Vis Sci 2008; 49: 772-780.

34. Liu J, Lu W, Guha S, Baltazar GC, Coffey EE, Laties AM et al. Cystic fibrosis transmembrane conductance regulator (CFTR) contributes to reacidification of alkalinized lysosomes in RPE cells. Am J Physiol Cell Physiol 2012; 303: C160-C169.

35. Guha S, Baltazar GC, Tu LA, Liu J, Lim JC, Lu W et al. Stimulation of the D5 dopamine receptor acidifies the lysosomal $\mathrm{pH}$ of retinal pigmented epithelial cells and decreases accumulation of autofluorescent photoreceptor debris. J Neurochem 2012; 122: 823-833.

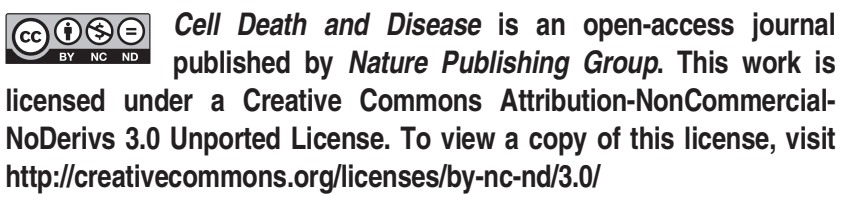

Cell Death and Disease is an open-access journal published by Nature Publishing Group. This work is http://creativecommons.org/licenses/by-nc-nd/3.0/ 